\title{
Plasma shaping effects on tokamak scrape-off layer turbulence
}

\author{
Fabio Riva, ${ }^{1, *}$ Emmanuel Lanti, ${ }^{1}$ Sébastien Jolliet, ${ }^{1}$ and Paolo Ricci ${ }^{1}$ \\ ${ }^{1}$ École Polytechnique Fédérale de Lausanne (EPFL), \\ Swiss Plasma Center (SPC), CH-1015 Lausanne, Switzerland
}

\begin{abstract}
The impact of plasma shaping on tokamak scrape-off layer (SOL) turbulence is investigated. The drift-reduced Braginskii equations are written for arbitrary magnetic geometries, and an analytical equilibrium model is used to introduce the dependence of turbulence equations on tokamak inverse aspect ratio $(\epsilon)$, Shafranov's shift $(\Delta)$, elongation $(\kappa)$, and triangularity $(\delta)$. A linear study of plasma shaping effects on the growth rate of resistive ballooning modes (RBMs) and resistive drift waves (RDWs) reveals that RBMs are strongly stabilized by elongation and negative triangularity, while RDWs are only slightly stabilized in non-circular magnetic geometries. Assuming that the linear instabilities saturate due to non-linear local flattening of the plasma gradient, the equilibrium gradient pressure length $L_{p}=-p_{e} / \nabla p_{e}$ in the SOL is numerically computed and its dependence on $\epsilon, \Delta, \kappa$, and $\delta$ is analyzed, showing that stabilization of RBMs results in shorter $L_{p}$. An analytical estimate of $L_{p}$ in the infinit aspect ratio limit and neglecting the Shafranov's shift is also derived. Non-linear SOL turbulence simulations with non-circular magnetic geometries are carried out using the global, three-dimensional, flux-driven fluid code GBS [P. Ricci et al., Plasma Phys. Controlled Fusion 54, 124047 (2012)] and the results are compared with the findings obtained from the linear analysis of the SOL instabilities, showing good quantitative agreement.
\end{abstract}

*Electronic address: fabio.riva@epfl.ch 


\section{INTRODUCTION}

The understanding of the turbulent processes occurring in the Scrape-Off Layer (SOL) of tokamaks is of fundamental importance for determining the performance of future fusion devices such as ITER. This region, characterized by magnetic field lines that intercept solid wall surfaces, establishes the boundary conditions for the plasma core, controls the fueling and impurity dynamics, determines the overall plasma confinement, and exhausts the tokamak power [1]. The heat load on the vessel of the device may become a show stopper of the fusion program if material constraints cannot be met.

The SOL plasma dynamics results from the interplay of perpendicular turbulent transport, driven by instabilities such as ballooning modes (BMs) and drift waves (DWs), destabilized by plasma gradients and unfavorable magnetic curvature, and the parallel losses at the end of the magnetic field lines [2-6]. This balance determines the SOL width and the heat load to the vessel.

In the past decade, several computational models were developed and implemented into numerical codes, that are used to investigate the tokamak SOL dynamics [7-16]. The main instabilities driving the SOL turbulence were characterized in the simplest SOL configuration (i.e. limited and caracterized by a circular magnetic equilibrium) [17-19], and a predictive first-principle model of the SOL width was derived and compared with experimental observations $[20,21]$. Despite the fact that the effects of plasma shaping on core turbulence have been extensively studied, both experimentally and numerically (see, e.g., Refs. [22-26]), the attemps to study the effects of plasma shaping on SOL turbulence in limited geometry are only experimental (see, e.g., Ref. [27]). This motivates the present work, where the impact of tokamak inverse aspect ratio $(\epsilon)$, Shafranov's shift $(\Delta)$, elongation $(\kappa)$, and triangularity $(\delta)$ on the SOL dynamics is studied. The goal of our analysis is twofold. First, the main linear instability driving the SOL dynamics is identified, depending on the shape of the magnetic geometry. Second, an analytical model for the characteristic equilibrium gradient pressure length, $L_{p}=-p_{e} / \nabla p_{e}$, that features shaping effects is derived.

Our study is based on the drift-reduced Braginskii equations [28, 29], which we express in arbitrary magnetic geometry. We focus on a limited SOL, this being the simplest configuration retaining the relevant effects of plasma shaping, and we use a simple analytical equilibrium model to express the dependence of the magnetic field on $\epsilon, \Delta, \kappa$, and $\delta$ [30]. 
The impact of the magnetic geometry on the growth rate of BMs and DWs is analyzed using a linearized model. Assuming that the linear instabilities saturate due to a non-linear local flattening of the plasma gradient and the resulting removal of the instability drive [31], we determine the main instability driving SOL turbulence and express $L_{p}$ as a function of the shaping parameters.

Our theoretical findings are compared with the results of three-dimensional, global, fluxdriven, non-linear simulations of SOL turbulence carried out with GBS $[15,16]$. This code implements the drift-reduced Braginskii equations with a set of boundary conditions describing the magnetic pre-sheath entrance [32], with no separation between equilibrium and fluctuations. Consequently, it provides quasi-steady state plasma profiles which are selfconsistently obtained from the balance between plasma sources, perpendicular transport, and parallel losses at the vessel. GBS has been extensively used to study the turbulent processes occurring in the SOL [17-19, 33-35], and its results were recently rigorously verified and validated against experimental measurements [36-39].

This paper is structured as follows. After the Introduction, in Sec. II we discuss the fluid model used to describe the SOL dynamics, the employed coordinate systems, and the magnetic equilibrium. Then, in Sec. III, we focus on the SOL linear instabilities, in particular, resistive BMs (RBMs) and resistive DWs (RDWs), and we illustrate the effects of plasma shaping on their growth rate. The presentation of the non-linear mechanism that regulates the amplitude of plasma turbulence is the subject of Sec. IV, where we derive a first-principle predictive scaling for the SOL width when non-circular magnetic equilibria are considered. Section $\mathrm{V}$ is devoted to the discussion of the non-linear simulations performed with GBS and their agreement with the results in Secs. III and IV. Finally, we present a possible physical interpretation of our results in Sec. VI and we report our conclusions in Sec. VII. The computation of the metric coefficients, the derivation of the magnetic equilibrium, and the analytical curvature operator used to obtain the $L_{p}$ analytical scaling, are illustrated in Appendices A, B, and C, respectively.

\section{THE MODEL}

The high plasma collisionality in the tokamak SOL allows neglecting kinetic effects and using a fluid approach to describe plasma dynamics $[12,29,40]$. Since fluctuations with 
amplitude and size comparable to the time-averaged values are observed [41], a flux-driven model is necessary to study SOL turbulence. In this section, we present the analytical model considered for the analysis of SOL turbulence, which is implemented in the GBS code.

\section{A. Fluid moment equations}

The analysis of the SOL dynamics, presented in this paper, is based on the use of the drift-reduced Braginskii equations [28, 29] in the electrostatic, cold ion, limit. Assuming that the time scale of the turbulence is such that $d / d t \ll \omega_{c i}$ (where $\omega_{c i}=e B / m_{i}$ is the ion gyrofrequency), the perpendicular velocities can be written as $\mathbf{v}_{\perp i}=\mathbf{v}_{E \times B}+\mathbf{v}_{\text {pol }}$ and $\mathbf{v}_{\perp e}=\mathbf{v}_{E \times B}+\mathbf{v}_{d e}$, where $\mathbf{v}_{E \times B}=-\nabla \phi \times \mathbf{B} / B^{2}$ is the $E \times B$ velocity, $\mathbf{v}_{\text {pol }}$ is the polarization velocity defined in Ref. [29], and $\mathbf{v}_{d e}=-\mathbf{B} \times \nabla p_{e} /\left(e n B^{2}\right)$ is the electron diamagnetic velocity. The resulting system of equations in normalized units writes

$$
\begin{aligned}
\frac{\partial n}{\partial t}= & -\frac{R_{0}}{B}\{\phi, n\}+\frac{2}{B}\left[C\left(p_{e}\right)-n C(\phi)\right]-\nabla \cdot\left(n v_{\| e} \mathbf{b}\right)+\mathcal{D}_{n}(n)+S_{n} \\
\frac{\partial \omega}{\partial t}= & -\frac{R_{0}}{B}\{\phi, \omega\}+\frac{2 B}{n} C\left(p_{e}\right)-v_{\| i} \nabla_{\|} \omega+\frac{B^{2}}{n} \nabla \cdot\left(j_{\|} \mathbf{b}\right)+\frac{B}{3 n} C\left(G_{i}\right)+\mathcal{D}_{\omega}(\omega) \\
\frac{\partial v_{\| e}}{\partial t}= & -\frac{R_{0}}{B}\left\{\phi, v_{\| e}\right\}+\frac{m_{i}}{m_{e}}\left[\nabla_{\|} \phi-\frac{\nabla_{\|} p_{e}}{n}-0.71 \nabla_{\|} T_{e}+\frac{\nu j_{\|}}{n}-\frac{2}{3 n} \nabla_{\|} G_{e}\right] \\
& -v_{\| e} \nabla_{\|} v_{\| e}+\mathcal{D}_{v_{\|} e}\left(v_{\| e}\right) \\
\frac{\partial v_{\| i}}{\partial t}= & -\frac{R_{0}}{B}\left\{\phi, v_{\| i}\right\}-v_{\| i} \nabla_{\|} v_{\| i}-\frac{\nabla_{\|} p_{e}}{n}-\frac{2}{3 n} \nabla_{\|} G_{i}+\mathcal{D}_{v_{\|} i}\left(v_{\| i}\right) \\
\frac{\partial T_{e}}{\partial t}= & -\frac{R_{0}}{B}\left\{\phi, T_{e}\right\}+\frac{4}{3 B} T_{e}\left[\frac{C\left(p_{e}\right)}{n}+\frac{5}{2} C\left(T_{e}\right)-C(\phi)\right]-v_{\| e} \nabla_{\|} T_{e}+S_{T_{e}} \\
& +\frac{2}{3} T_{e}\left[0.71 \frac{\nabla \cdot\left(j_{\|} \mathbf{b}\right)}{n}-\nabla \cdot\left(v_{\| e} \mathbf{b}\right)\right]+\mathcal{D}_{T_{e}}\left(T_{e}\right)+\mathcal{D}_{\|, T_{e}}\left(T_{e}\right),
\end{aligned}
$$

where $m_{i} / m_{e}$ is the ion to electron mass ratio, $B$ is the norm of the magnetic field, $\mathbf{b}=\mathbf{B} / B$ is the magnetic field unit vector, $R_{0}$ is the normalized major radius, $j_{\|}=n\left(v_{\| i}-v_{\| e}\right)$ is the parallel current, $p_{e}=n T_{e}$ is the electron plasma pressure, $\omega=\nabla_{\perp}^{2} \phi$ is the vorticity, and $\nu$ is the normalized Spitzer resistivity. The density and electron temperature sources $S_{n}$ and $S_{T_{e}}$ are used to mimic the plasma outflow from the core. The expressions of the two terms representing the ion and electron gyroviscous contributions are respectively given by $G_{i}=-\eta_{0 i}\left[2 \nabla_{\|} v_{\| i}+C(\phi) / B\right]$ and $G_{e}=-\eta_{0 e}\left[2 \nabla_{\|} v_{\| e}-C\left(p_{e}\right) /(n B)+C(\phi) / B\right]$, where 
$\eta_{0 i}$ and $\eta_{0 e}$ are the gyroviscous coefficients [28]. The Poisson brackets are defined as $\{\phi, A\}=$ $\mathbf{b} \cdot(\nabla \phi \times \nabla A)$, the curvature operator as $C(A)=B / 2[\nabla \times(\mathbf{b} / B)] \cdot \nabla A$, the parallel gradient as $\nabla_{\|} A=\mathbf{b} \cdot \nabla A$, and the perpendicular Laplacian as $\nabla_{\perp}^{2} A=-\nabla \cdot[\mathbf{b} \times(\mathbf{b} \times \nabla A)]$, with $A=n, \omega, \phi, v_{\| i}, v_{\| e}, T_{e}$. We note that the Boussinesq's approximation [42-44] is used in the evaluation of the divergence of the polarization current in Eq. (2). Small perpendicular and parallel diffusion terms of the form $\mathcal{D}_{A}(A)=D_{A} \nabla_{\perp}^{2} A$ and $\mathcal{D}_{\|, A}(A)=\chi_{\|, A} \nabla_{\|}^{2} A$ are added for the numerical solution of Eqs. (1)-(5). In the present paper, all quantities are normalized according to (tilde denotes a physical quantity in SI units): $t=\tilde{t} /\left(\tilde{R} / \tilde{c}_{s 0}\right)$, $n=\tilde{n} / \tilde{n}_{0}, T_{e}=\tilde{T}_{e} / \tilde{T}_{e 0}, \phi=e \tilde{\phi} / \tilde{T}_{e 0}, v_{\| e}=\tilde{v}_{\| e} / \tilde{c}_{s 0}, v_{\| i}=\tilde{v}_{\| i} / \tilde{c}_{s 0}, B=\tilde{B} / \tilde{B}_{0}, R_{0}=\tilde{R} / \tilde{\rho}_{s 0}$, $\nu=\left(e^{2} \tilde{n}_{0} \tilde{R}\right) /\left(m_{i} \tilde{\sigma}_{\|} \tilde{c}_{s 0}\right)$, where $\tilde{\sigma}_{\|}$is the parallel conductivity, $\tilde{n}_{0}, \tilde{T}_{e 0}$ and $\tilde{B}_{0}$ are reference density, temperature, and magnetic field, $\tilde{R}$ is the tokamak major radius, and $\tilde{c}_{s 0}$ and $\tilde{\rho}_{s 0}$ are given by $\tilde{c}_{s 0}=\sqrt{\tilde{T}_{e 0} / m_{i}}$ and $\tilde{\rho}_{s 0}=\tilde{c}_{s 0} m_{i} /\left(e \tilde{B}_{0}\right)$. Distances perpendicular to $\mathbf{B}$ are normalized to $\tilde{\rho}_{s 0}$, while parallel distances are normalized to $\tilde{R}$.

Equations (1)-(5) are completed by a set of boundary conditions at the magnetic pre-sheath entrance, where the validity of the drift approximation breaks down [32]. In normalized units, and neglecting the contribution of the radial gradients, these boundary conditions are given by

$$
\begin{aligned}
v_{\| i} & = \pm c_{s} \\
v_{\| e} & = \pm c_{s} \exp \left(\Lambda-\phi / T_{e}\right) \\
\partial_{s} T_{e} & =0 \\
\partial_{s} n & =\mp \frac{n}{c_{s}} \partial_{s} v_{\| i} \\
\omega & =-\left(\partial_{s} v_{\| i}\right)^{2} \mp c_{s} \partial_{s}^{2} v_{\| i} \\
\partial_{s} \phi & =\mp c_{s} \partial_{s} v_{\| i},
\end{aligned}
$$

where the upper (lower) sign refers to the case of a magnetic field directed toward (away from) the wall, $c_{s}=\sqrt{T_{e}}$ is the ion sound speed, $\Lambda=\log \sqrt{m_{i} /\left(2 \pi m_{e}\right)} \simeq 3$ for hydrogen plasmas, and $s$ is a coordinate normal to the wall, which coincides with the poloidal angle in the infinite aspect ratio limit. 


\section{B. Coordinate systems and differential operators}

We express here Eqs. (1)-(5) for arbitrary magnetic geometries. We note that the dependence on the magnetic field geometry enters in the model through: (i) the norm of the magnetic field $B$, (ii) the direction of the unit vector $\mathbf{b}$, and (iii) the differential operators $\{\phi,-\}, C(-), \nabla_{\|}(-)$, and $\nabla_{\perp}^{2}(-)$, which are computed, having defined a magnetic geometry, by expressing the covariant and contravariant components of the magnetic field and of the metric tensor in the chosen coordinate system.

In the present paper, we make use of the toric $\left(\theta_{*}, r, \varphi\right)$ and the flux-tube $\left(r, \alpha, \theta_{*}\right)$ coordinate systems, where $r$ is a flux coordinate, $\varphi$ is the toroidal angle, $\alpha=\varphi-q(r) \theta_{*}$ is a field line label, and $\theta_{*}$ is the straight field line angle defined as

$$
\theta_{*}(r, \theta)=\frac{1}{q(r)} \int_{0}^{\theta} \frac{\mathbf{B} \cdot \nabla \varphi}{\mathbf{B} \cdot \nabla \theta^{\prime}} \mathrm{d} \theta^{\prime}
$$

with $\theta$ and $\theta^{\prime}$ the poloidal angle and

$$
q(r)=\frac{1}{2 \pi} \int_{0}^{2 \pi} \frac{\mathbf{B} \cdot \nabla \varphi}{\mathbf{B} \cdot \nabla \theta} \mathrm{d} \theta
$$

the safety factor. We remark that, in the remainder of this paper, we rescale the toric coordinate system as $y=a \theta_{*}, x=r$, and $z=R_{0} \varphi$, where $a$ is the tokamak minor radius in $\tilde{\rho}_{s 0}$ units. We also rescale the flux-tube coordinate system as $X=r, Y=(a / q) \alpha$, and $Z=q R_{0} \theta_{*}$, with $q=q(a)$ the safety factor at the last close flux surface (LCFS).

In the $(y, x, z)$ coordinate system, the differential operators can be written in their advection form, obtaining

$$
\begin{aligned}
\{\phi, A\} & =\mathcal{P}^{y x}\{\phi, A\}_{y x}+\mathcal{P}^{x z}\{\phi, A\}_{x z}+\mathcal{P}^{z y}\{\phi, A\}_{z y} \\
\nabla_{\|} A & =\mathcal{D}^{y} \frac{\partial A}{\partial y}+\mathcal{D}^{x} \frac{\partial A}{\partial x}+\mathcal{D}^{z} \frac{\partial A}{\partial z} \\
C(A) & =\mathcal{C}^{y} \frac{\partial A}{\partial y}+\mathcal{C}^{x} \frac{\partial A}{\partial x}+\mathcal{C}^{z} \frac{\partial A}{\partial z} \\
\nabla_{\perp}^{2} A & =\mathcal{N}^{y y} \frac{\partial^{2} A}{\partial y^{2}}+\mathcal{N}^{y x} \frac{\partial^{2} A}{\partial y \partial x}+\mathcal{N}^{x x} \frac{\partial^{2} A}{\partial x^{2}}+\mathcal{N}^{y} \frac{\partial A}{\partial y}+\mathcal{N}^{x} \frac{\partial A}{\partial x} \\
& +\mathcal{N}^{z z} \frac{\partial^{2} A}{\partial z^{2}}+\mathcal{N}^{x z} \frac{\partial^{2} A}{\partial x \partial z}+\mathcal{N}^{y z} \frac{\partial^{2} A}{\partial y \partial z}+\mathcal{N}^{z} \frac{\partial A}{\partial z}
\end{aligned}
$$

with $\{\phi, A\}_{y x}=\partial_{x} A \partial_{y} \phi-\partial_{y} A \partial_{x} \phi$, while equivalent expressions are found considering the $(X, Y, Z)$ coordinate system. Assuming an axisymmetric magnetic field and that turbulence is characterized by $\nabla_{\perp} A / \nabla_{\|} A \gg 1$, one can compute the coefficients in Eqs. (14)-(17) (the 
details of the derivation are presented in Appendix A). The resulting non-zero coefficients are

$$
\begin{aligned}
& \mathcal{P}^{y x}=\frac{a}{\mathcal{J} b_{\varphi}}, \quad \mathcal{C}^{x}=-\frac{R_{0} B}{2 \mathcal{J}} \frac{\partial c_{\varphi}}{\partial \theta_{*}}, \quad \mathcal{C}^{y}=\frac{a R_{0} B}{2 \mathcal{J}}\left[\frac{\partial c_{\varphi}}{\partial r}+\frac{1}{q}\left(\frac{\partial c_{\theta_{*}}}{\partial r}-\frac{\partial c_{r}}{\partial \theta_{*}}\right)\right] \\
& \mathcal{N}^{x x}=g^{r r}, \quad \mathcal{N}^{x y}=2 a g^{r \theta_{*}}, \quad \mathcal{N}^{y y}=a^{2}\left(g^{\theta_{*} \theta_{*}}+\frac{g^{\varphi \varphi}}{q^{2}}\right) \\
& \mathcal{N}^{x}=\nabla^{2} r, \quad \mathcal{N}^{y}=a \nabla^{2} \theta_{*}
\end{aligned}
$$

for the $(y, x, z)$ coordinate system and

$$
\begin{aligned}
& \mathcal{P}^{X Y}=-\frac{b_{\theta_{*}} a}{\mathcal{J} q}, \quad \quad \mathcal{D}^{Z}=q R_{0} b^{\theta_{*}} \\
& \mathcal{C}^{X}=-\frac{R_{0} B}{2 \mathcal{J}} \frac{\partial c_{\alpha}}{\partial \theta_{*}}, \quad \mathcal{C}^{Y}=\frac{a R_{0} B}{2 \mathcal{J} q}\left(\frac{\partial c_{r}}{\partial \theta_{*}}-\frac{\partial c_{\theta_{*}}}{\partial r}\right) \\
& \mathcal{N}^{X X}=g^{r r}, \quad \mathcal{N}^{X Y}=\frac{2 g^{\alpha r} a}{q}, \quad \mathcal{N}^{Y Y}=\frac{a^{2} g^{\alpha \alpha}}{q^{2}} \\
& \mathcal{N}^{X}=\nabla^{2} r, \quad \mathcal{N}^{Y}=\frac{a}{q} \nabla^{2} \alpha
\end{aligned}
$$

for the $(X, Y, Z)$ coordinate system.

\section{Magnetic equilibrium}

In the present paper, we consider the magnetic equilibrium that is obtained by solving the Grad-Shafranov equation in the $r / R_{0} \rightarrow 0$ limit, taking into account elongation and non-zero triangularity [30], neglecting the plasma pressure contribution, and extrapolating the equilibrium to $r=a$. The solution of the Grad-Shafranov equation, presented in Appendix B, leads to

$$
\begin{aligned}
R_{c}(r, \theta) & =R_{0}\left\{1+\frac{r}{R_{0}} \cos \theta+\frac{\Delta(r)}{R_{0}}+\sum_{m=2}^{3} \frac{S_{m}(r)}{R_{0}} \cos [(m-1) \theta]-\frac{P(r)}{R_{0}} \cos \theta\right\} \\
Z_{c}(r, \theta) & =R_{0}\left\{\frac{r}{R_{0}} \sin \theta-\sum_{m=2}^{3} \frac{S_{m}(r)}{R_{0}} \sin [(m-1) \theta]-\frac{P(r)}{R_{0}} \sin \theta\right\} \\
F(r) & =R_{0}\left\{1-\frac{r^{2}}{R_{0}^{2}} \frac{1}{q(r)^{2}}\left[2+\frac{q-q_{0}}{q_{0}}\left(\frac{r}{a}\right)^{2}\right]\right\} \\
\psi^{\prime}(r) & =\frac{F(r)}{2 \pi q(r)} \int_{0}^{2 \pi} \frac{\mathcal{J}_{\theta r \varphi}}{R_{c}(r, \theta)^{2}} \mathrm{~d} \theta \\
q(r) & =q_{0}+\left(q-q_{0}\right)\left(\frac{r}{a}\right)^{2},
\end{aligned}
$$



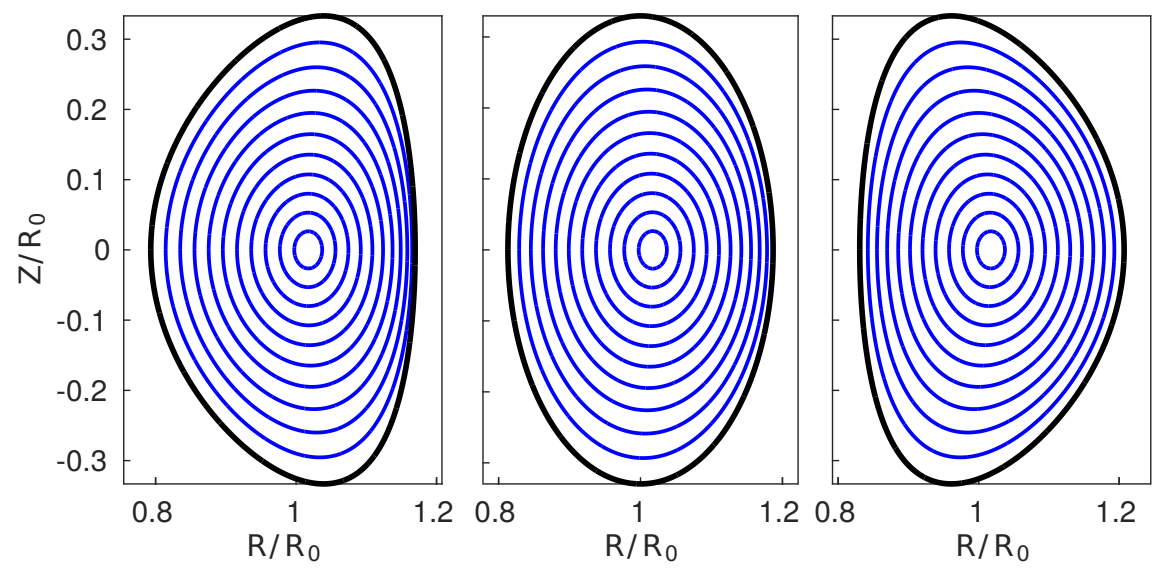

FIG. 1: Poloidal cuts of the magnetic surfaces generated assuming a magnetic equilibrium given by Eqs. (25)-(29), for $\epsilon=0.25, \Delta(0) \simeq 7$ (in $\tilde{\rho}_{s 0}$ units and for $\beta_{p}=0$ ), and $\kappa=1.8$, and $\delta=-0.3$ (left), $\delta=0$ (middle), and $\delta=0.3$ (right). Black thick lines represent the LCFS, while blue lines represent magnetic surfaces in the core.

where $\Delta(r)$ is the Shafranov's shift in the $\beta_{p} \rightarrow 0$ limit (being $\beta_{p}$ the ratio of the plasma pressure to the poloidal magnetic pressure), $S_{2}(r)$ and $S_{3}(r)$ are shaping functions related to $\kappa$ and $\delta$ by $\kappa=\left[a-S_{2}(a)\right] /\left[a+S_{2}(a)\right]$ and $\delta=4 S_{3}(a) / a, q_{0}$ is the safety factor at the magnetic axis, and $\mathcal{J}_{\theta r \varphi}=R_{c}\left(\partial_{r} R_{c} \partial_{\theta} Z_{c}-\partial_{\theta} R_{c} \partial_{r} Z_{c}\right)$ is the Jacobian associated with the $(\theta, r, \varphi)$ coordinate system. The analytical expressions of the functions $S_{2}(r), S_{3}(r), \Delta^{\prime}(r)$, and $P(r)$ are given in Appendix B. An example of the magnetic surfaces resulting from this geometry is presented in Fig. 1.

Combining Eq. (12) with the expression of the axisymmetric field $\mathbf{B}$, one obtains

$$
\theta_{*}(r, \theta)=\frac{F(r)}{q(r) \psi^{\prime}(r)} \int_{0}^{\theta} \frac{\mathcal{J}_{\theta^{\prime} r \varphi}}{R_{c}(r, \theta)^{2}} \mathrm{~d} \theta^{\prime} .
$$

We note that $\theta_{*}=\theta=0$ and $\theta_{*}=\theta=2 \pi$ correspond to the equatorial low-field side midplane and that, in general, Eq. (30) is not analytically integrable, but it is possible to find numerically the two functions $\theta_{*}=\theta_{*}(r, \theta)$ and the inverse $\theta=\theta\left(r, \theta_{*}\right)$. From these, all the coefficients of Eqs. (18)-(24) can be computed, and Eqs. (1)-(5) are completely defined. Finally, we note that in the limit $\Delta(0) \rightarrow 0, \kappa \rightarrow 1, \delta \rightarrow 0$, and neglecting $P(r)$ and the $r^{2} / R_{0}^{2}$ term of Eq. (27), we obtain the circular magnetic equilibrium discussed in Ref. [18]. 


\section{LINEAR INSTABILITIES}

The turbulent transport observed in the tokamak SOL is due to the non-linear development of linear modes that are destabilized by plasma gradients and unfavorable magnetic curvature in the presence of resistivity and electron inertia [2-6]. For typical SOL parameters, RBMs and RDWs are found to be the main instabilities driving plasma turbulence $[11,17,45]$.

The reminder of this section is devoted to the discussion of the properties of SOL linear instabilities and, in particular, to the derivation of RBMs and RDWs dispersion relations in non-circular magnetic geometries. First, Eqs. (1)-(5) are expressed in the $(X, Y, Z)$ coordinate system and the resulting system of equations is linearized assuming that the equilibrium plasma profiles depend only on the radial coordinate $X$. Each quantity $A=A(X, Y, Z, t)$ is then split between an equilibrium part $A_{0}(X)$ and the perturbation $\delta A(Y, Z, t)=\delta A(Z) \exp \left[i k_{Y} Y+\gamma t\right]$, with $k_{Y}$ the poloidal wave number and $\gamma$ the linear growth rate. Equilibrium gradients are defined as $\partial_{X} A=-A_{0} / L_{A}$, where $L_{A}$ is a characteristic length associated with $A_{0}$ at $X=a$. The $X$ dependence of $\delta A$ is neglected here because for both DWs and BMs $k_{Y} / k_{X} \sim \sqrt{k_{Y} L_{n}}>1[46,47]$. Assuming $\phi_{0}=v_{\| i, 0}=v_{\| e, 0}=0$, noting that $n_{0}=1$ and $T_{e 0}=1$ in normalized units, and neglecting gyroviscous and diffusion terms, the resulting system of equations writes

$$
\begin{aligned}
\gamma \delta n & =\frac{R_{0}}{L_{n}} \frac{1}{B} \mathcal{P}^{L}(\delta \phi)+\frac{2}{B} \mathcal{C}^{L}\left(\delta p_{e}-\delta \phi\right)+\left(\nabla_{\|}+\nabla \cdot \mathbf{b}\right)\left(\delta j_{\|}-\delta v_{\| i}\right) \\
\frac{1}{B^{2}} \gamma \delta \omega & =\frac{2}{B} \mathcal{C}^{L}\left(\delta p_{e}\right)+\left(\nabla_{\|}+\nabla \cdot \mathbf{b}\right) \delta j_{\|} \\
\frac{m_{e}}{m_{i}} \gamma \delta v_{\| e} & =\nabla_{\|}\left(\delta \phi-\delta p_{e}-0.71 \delta T_{e}\right)+\nu \delta j_{\|} \\
\gamma \delta v_{\| i} & =-\nabla_{\|} \delta p_{e} \\
\gamma \delta T_{e} & =\frac{R_{0}}{L_{n}} \frac{\eta}{B} \mathcal{P}^{L}(\delta \phi)+\frac{4}{3 B} \mathcal{C}^{L}\left(\delta p_{e}+\frac{5}{2} \delta T_{e}-\delta \phi\right)+\frac{2}{3}\left(\nabla_{\|}+\nabla \cdot \mathbf{b}\right)\left(1.71 \delta j_{\|}-\delta v_{\| i}\right)
\end{aligned}
$$

where $\delta p_{e}=\delta n+\delta T_{e}, \delta j_{\|}=\delta v_{\| i}-\delta v_{\| e}, \delta \omega=\left(\nabla_{\perp}^{2}\right)^{L} \delta \phi, \eta=L_{n} / L_{T_{e}}$ and

$$
\mathcal{P}^{L}(A)=i \mathcal{P}^{X Y} k_{Y} A, \quad \mathcal{C}^{L}(A)=i \mathcal{C}^{Y} k_{Y} A, \quad\left(\nabla_{\perp}^{2}\right)^{L} A=-\mathcal{N}^{Y Y} k_{Y}^{2} A
$$

Equations (31)-(35) determine the linear growth rate of SOL plasma instabilities. Plasma shaping affects the growth rate through four terms: $\nabla_{\|}(-)+\nabla \cdot \mathbf{b}$ that results from the 
plasma advection along magnetic field lines, $\mathcal{P}^{L}(-) / B$ that represents the $E \times B$ convection, $\mathcal{C}^{L}(-) / B$ that introduces curvature drifts and plasma compressibility, and $\left(\nabla_{\perp}^{2}\right)^{L}(-) / B^{2}$, the vorticity operator.

To solve Eqs. (31)-(35) in arbitrary magnetic geometry, a numerical code was developed, which evaluates $\gamma$ as a function of the parameters $R_{0} / L_{n}, \eta, \nu, q, k_{Y}, \epsilon, \kappa$, and $\delta$, with $\epsilon=a / R_{0}$. The numerical implementation of the code is detailed in Ref. [48], and its main features are summarized here. First, the $Z$ component is discretized using a fourth order finite difference scheme. Second, Dirichlet boundary conditions are imposed to $\delta n, \delta \phi$, and $\delta T_{e}$, while no boundary conditions are applied to the ion and electron parallel velocities (the impact of the boundary conditions on the growth rate of the instability is negligible when considering RBMs and RDWs and a limitier at the inner midplane [18]). Finally, the discretized system of equations is integrated implicitly in time starting from random noise. By studying the growth of the most unstable mode, we obtain $\gamma$.

To investigate the influence of plasma shape on the linear instabilities, the coefficients of the differential operators are implemented according to Eqs. (21)-(24) at $X=a$. In particular, Eq. (30) is solved numerically, using the trapezoidal rule to approximate the integral. Then, the relation $\theta_{*}=\theta_{*}(r, \theta)$ is inverted using a linear interpolation scheme, to obtain the function $\theta=\theta\left(r, \theta_{*}\right)$. The derivatives of the magnetic field components and of the metric coefficients appearing in Eqs. (21)-(24) are then computed using second order finite difference schemes. We verified that the evaluation of the geometric coefficients is numerically converged, and that, in the limit of circular magnetic surfaces, we recover the analytical expressions given in Ref. [18].

Previous investigations of SOL instabilities show that the effects of electron inertia can be neglected with respect to resistivity in typical SOL conditions [17]. The inertia term on left-hand side of Eq. (33) can thus be neglected. In the following, we focus our attention on this high plasma resistivity regime by setting $\nu=0.1$ and describe the impact of plasma shaping on RBMs and RDWs, which are the main SOL instabilities. This value is larger than in usual tokamaks SOL plasma conditions, and it is chosen to completely decouple the restistive and the inertial branches of the BMs and DWs. As shown in the remainder of this paper, plasma shaping mainly influences the RBMs growth rate, while the value of $\nu$ has only a minor impact in determining the linear growth rate and the wave number of the mode driving the turbulent transport. Note that we consider $q=4, R_{0}=500, \hat{s}(a)=0$, 
and $\eta=0.66$ for our linear studies ( $\eta$ is obtained from non-linear simulations, see Sec. V, in agreement with Ref. [47] results). The small positive value of the magnetic shear in limited discharges is expected to stabilize DWs $[46,48]$ and should weakly affect BMs.

\section{A. Resistive ballooning modes}

Resistive BMs are interchange-like modes, driven unstable in the presence of finite resistivity when the plasma pressure gradient and the magnetic field line curvature point in the same direction [49-53]. Setting $\partial_{Z} \rightarrow i k_{Z}$ and neglecting the compressibility terms with respect to the advection terms, the parallel dynamics in the continuity and temperature equations, the diamagnetic term in the Ohm's law to avoid coupling with DWs, as well as the coupling with sound waves (valid for $\gamma \gg k_{Z}, k_{Z} \sim 1 / q$ for RBMs), Eqs. (31)-(35) can be simplified to obtain

$$
\begin{aligned}
\gamma \delta p_{e} & =\frac{R_{0}}{L_{p}} \frac{1}{B} \mathcal{P}^{L}(\delta \phi) \\
\frac{1}{B^{2}} \gamma\left(\nabla_{\perp}^{2}\right)^{L} \delta \phi & =\frac{2}{B} \mathcal{C}^{L}\left(\delta p_{e}\right)+\left(i \mathcal{D}^{Z} k_{Z}+\nabla \cdot \mathbf{b}\right) \delta j_{\|} \\
0 & =\nu \delta j_{\|}+i \mathcal{D}^{Z} k_{Z} \delta \phi
\end{aligned}
$$

where $L_{p}=L_{n} /(1+\eta)$. Equations (37)-(39) constitute the minimal model describing the linear properties of RBMs.

Considering Eqs. (37)-(39) in a circular magnetic geometry in the $\epsilon \rightarrow 0$ limit, it was shown [48] that the peak growth rate, $\gamma \sim \gamma_{b}$, with $\gamma_{b}=\sqrt{2 R_{0} / L_{p}}$ being the ideal BM growth rate, occurs for $k_{Y} \sim k_{b}$, where $k_{b}=1 / \sqrt{q^{2} \nu \gamma_{b}}$. Imposing $k_{Y}=k_{b}$, we compute the ratio $\gamma / \gamma_{b}$ as a function of $\kappa$ and $\delta$ for $\epsilon \simeq 0.25$ and $\Delta(0) \simeq 7$. The results are presented in Fig. 2 (left panel). We observe that RBMs are stabilized for $\kappa>1$ and for $\delta<0$, while their growth rate is enhanced for $\delta>0.2$. To isolate the different shaping effects on the linear growth rate, we perform the same analysis for $\epsilon=0$ and $\Delta(0)=0$. The results are presented in Fig. 2 (right panel). While the same trends are recovered, the ratio $\gamma / \gamma_{b}$ is larger for $\epsilon=0$ and $\Delta(0)=0$ than for $\epsilon \simeq 0.25$ and $\Delta(0) \simeq 7$. This suggests that aspect ratio and Shafranov's shift effects stabilize RBMs, in agreement with the observations in Ref. [18]. Note that these results are independent of $L_{p}$.

A detailed analysis of the linear growth rate shows that the curvature operator affects the RBMs growth rate the most. As a matter of fact, assuming $\mathcal{P}^{X Y}=-1, \mathcal{N}^{Y Y}=1, \mathcal{D}^{Z}=1$, 

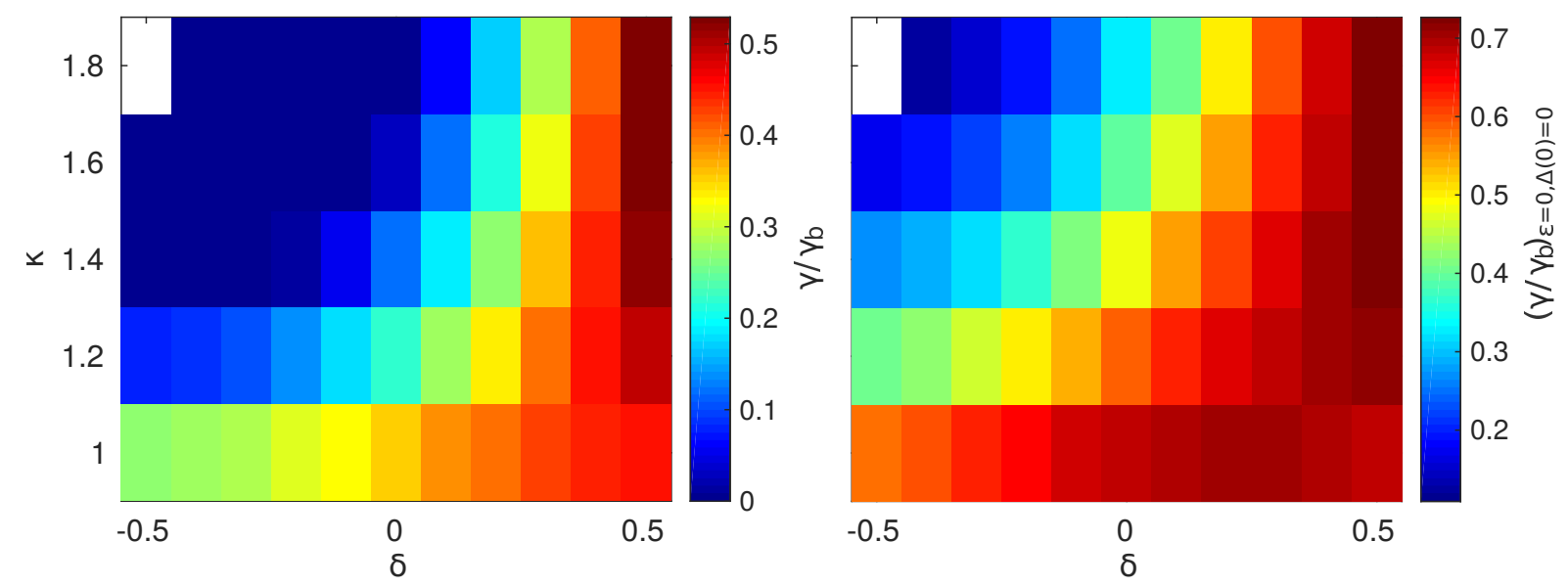

FIG. 2: Value of $\gamma / \gamma_{b}$ as a function of $\kappa$ and $\delta$, obtained considering RBMs and solving Eqs. (37)(39) for $k_{Y}=k_{b}$. The left panel is obtained for $\epsilon \simeq 0.25$ and $\Delta(0) \simeq 7$, while the right panel is computed for $\epsilon=0$ and $\Delta(0)=0$.

$\nabla \cdot \mathbf{b}=0, B=1$, and $\mathcal{C}^{Y}=-\left.\partial_{r} R(r, \theta)\right|_{\theta_{*}}$, where $\left.\right|_{\theta_{*}}$ indicates that the $r$ derivative is computed at fixed $\theta_{*}$, the results of Fig. 2 are recovered, within an error on $\gamma / \gamma_{b}$ typically less than $20 \%$.

This observation allows to simplify Eqs. (37)-(39) assuming a strongly ballooned mode around $\theta_{*}=0, k_{Z}=1 / q, \mathcal{P}^{X Y}=-1, \mathcal{N}^{Y Y}=1, \mathcal{D}^{Z}=1, \nabla \cdot \mathbf{b}=0, B=1$, and $\mathcal{C}^{Y}=-\left.\partial_{r} R(r, \theta)\right|_{\theta_{*}=0}$. In fact, one can write the RBMs dispersion relation as

$$
\bar{\gamma}^{2}+2 \gamma_{D} \bar{\gamma}-\gamma_{I}^{2}=0
$$

where $\gamma_{D}=-1 /\left(2 \nu q^{2} k_{Y}^{2} \gamma_{b}\right), \gamma_{I}=\sqrt{\left.\partial_{r} R(r, \theta)\right|_{\theta_{*}=0}}$, and $\bar{\gamma}=\gamma / \gamma_{b}$. Equation (40) shows that the RBM has a growth rate $\gamma=\gamma_{I} \gamma_{b}$ and it is stabilized by finite $k_{Z}$ effects through the $\gamma_{D}$ term.

\section{B. Resistive drift waves}

Resistive DWs are instabilities driven by the $E \times B$ plasma convection and destabilized when electron adiabaticity is broken by finite resistivity [54-58]. Assuming $\gamma \gg k_{Z}$ as for the RBMs, we can neglect sound wave coupling in Eqs. (31)-(35). Moreover, the curvature term in the vorticity equation can be neglected (to avoid coupling with BMs), together with the compressibility terms in the continuity and temperature equations. Consequently, we 

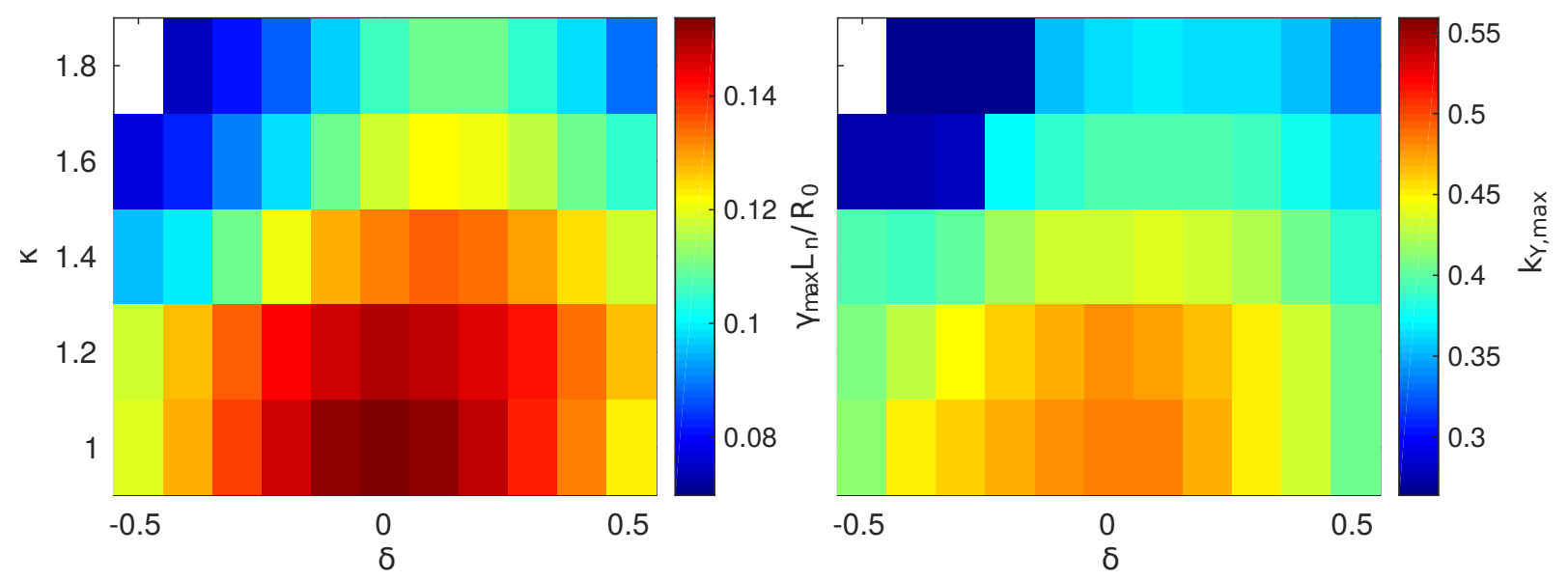

FIG. 3: Values of $\gamma_{\max } L_{n} / R_{0}$ (left panel) and $k_{Y, \max }$ (right panel) as a function of $\kappa$ and $\delta$, obtained considering RDWs and solving Eqs. (41)-(44) for $L_{p}=10, \epsilon \simeq 0.25$, and $\Delta(0) \simeq 7$.

obtain a reduced system of equations describing the RDWs dynamics, which writes

$$
\begin{aligned}
\gamma \delta n & =\frac{R_{0}}{L_{n}} \frac{1}{B} \mathcal{P}^{L}(\delta \phi)+\left(i \mathcal{D}^{Z} k_{Z}+\nabla \cdot \mathbf{b}\right) j_{\|} \\
\frac{1}{B^{2}} \gamma \delta \omega & =\left(i \mathcal{D}^{Z} k_{Z}+\nabla \cdot \mathbf{b}\right) \delta j_{\|} \\
0 & =i \mathcal{D}^{Z} k_{Z}\left(\delta \phi-\delta p_{e}-0.71 \delta T_{e}\right)+\nu \delta j_{\|} \\
\gamma \delta T_{e} & =\frac{R_{0}}{L_{n}} \frac{\eta}{B} \mathcal{P}^{L}(\delta \phi)+1.71 \frac{2}{3}\left(i \mathcal{D}^{Z} k_{Z}+\nabla \cdot \mathbf{b}\right) \delta j_{\|} .
\end{aligned}
$$

Equations (41)-(44) are solved as a function of $k_{Y}, \kappa$, and $\delta$, by using the linear solver previously described. For each magnetic shape, the growth rate is maximized over all possible $k_{Y}$ values, and the maximum value $\gamma_{\max }$ and the corresponding $k_{Y}$, noted $k_{Y, \max }$, are presented in Fig. 3 for $\epsilon \simeq 0.25$ and $\Delta(0) \simeq 7$. We note that $\gamma_{\max }$ normalized to $R_{0} / L_{n}$, i.e. $\gamma_{\max } L_{n} / R_{0}$, is independent of $L_{n}$. We see that $\gamma_{\max }$ associated with RDWs decreases both by increasing $\kappa$ and $|\delta|$, suggesting that RDWs are most unstable in circular magnetic geometry. However, the effect of plasma shaping on the RDW's growth rate is considerably weaker than on RBM's. We also note that $k_{Y, \max }$ decreases with $\kappa$ and $|\delta|$.

The analysis of the impact of aspect ratio and Shafranov's shift effects on RDWs growth rate shows a small influence on $\gamma_{\max }$ and $k_{Y \text {,max }}$, in agreement with the observations in Ref. [18]. Finally, we note that, considering a circular magnetic geometry in the infinite aspect ratio limit and setting $\partial_{Z} \rightarrow i k_{Z}$, we can simplify Eqs. (41)-(44) to write the dispersion relation 
of RDWs as

$$
\frac{1}{2} \hat{\gamma}^{2}+\alpha b \hat{\gamma}+i \alpha=0
$$

where $\alpha=k_{Z}^{2} L_{n} /\left[2 k_{Y} R_{0} \nu(1+1.71 \eta)\right], b=1 / k_{Y}+k_{Y}\left(1+1.71^{2} \cdot 2 / 3\right)$, and $\hat{\gamma}=\gamma L_{n} /\left[R_{0}(1+\right.$ 1.71) ]. We remark that Eq. (45) has to be solved over all the possible $k_{Y}$ and $k_{Z}$ values. In fact, $k_{Z}$ is a priori unknown for RDWs and depends on $\nu, R_{0} / L_{n}$, and $\eta$. Solving Eq. (45) numerically, it results that $\operatorname{Re}\left(\hat{\gamma}_{\max }\right) \simeq 0.0874$ for $k_{Y} \simeq 0.582$ and $\alpha \simeq 0.0412$, where $\operatorname{Re}(A)$ indicates the real part of $A$.

\section{ESTIMATE OF THE EQUILIBRIUM PRESSURE GRADIENT LENGTH}

The time-averaged plasma pressure gradient scale length $L_{p}$ in the tokamak SOL originates from a balance between the turbulent perpendicular transport of particles and heat, resulting from the non-linear development of the unstable modes, and the parallel losses at the end of the magnetic field lines. In the limit of negligible $E \times B$ shear flows and for typical SOL parameters, we assume that the gradient removal turbulence saturation mechanism, i.e. the local non-linear flattening of the plasma pressure profile and the resulting removal of the instability drive, is the mechanism that regulates the amplitude of SOL turbulence [31]. Our estimates of $L_{p}$ based on the gradient removal theory in circular magnetic flux surface geometry show agreement with non-linear simulations and experimental observations [20, 21]. The main features of the theory are briefly summarized here. The fundamental hypothesis is that the saturation of the growth rate of the linear modes occurs when these are able to remove their own drive, namely, the amplitude of the gradient associated with the fluctuation, $k_{X} \delta p_{e}$, is comparable to the gradient of the background pressure, $p_{e 0} / L_{p}$

$\left(k_{X} \sim \sqrt{k_{Y} / L_{p}}\right.$ is the radial extension of BMs or DWs obtained from a non-local linear theory $[46,47])$. Then, the leading terms in the pressure continuity equation provide an estimate of the electromagnetic potential fluctuations and therefore of the turbulent $E \times B$ flux, $\Gamma=k_{Y} \delta \phi \delta p_{e} \sim \gamma p_{e 0} /\left(R_{0} k_{Y}\right)$. Finally, the balance between the perpendicular turbulent transport, $R_{0} \partial_{X} \Gamma \sim R_{0} \Gamma / L_{p} \sim \gamma p_{e 0} /\left(k_{Y} L_{p}\right)$, and the parallel losses at the end of magnetic field lines, $\nabla_{\|}\left(p_{e} v_{\| e}\right) \sim p_{e 0} c_{s} / q$, gives $\gamma p_{e 0} /\left(k_{Y} L_{p}\right) \sim p_{e 0} c_{s} / q$, i.e.

$$
L_{p}=\frac{q}{c_{s}}\left(\frac{\gamma}{k_{Y}}\right)_{\max }
$$



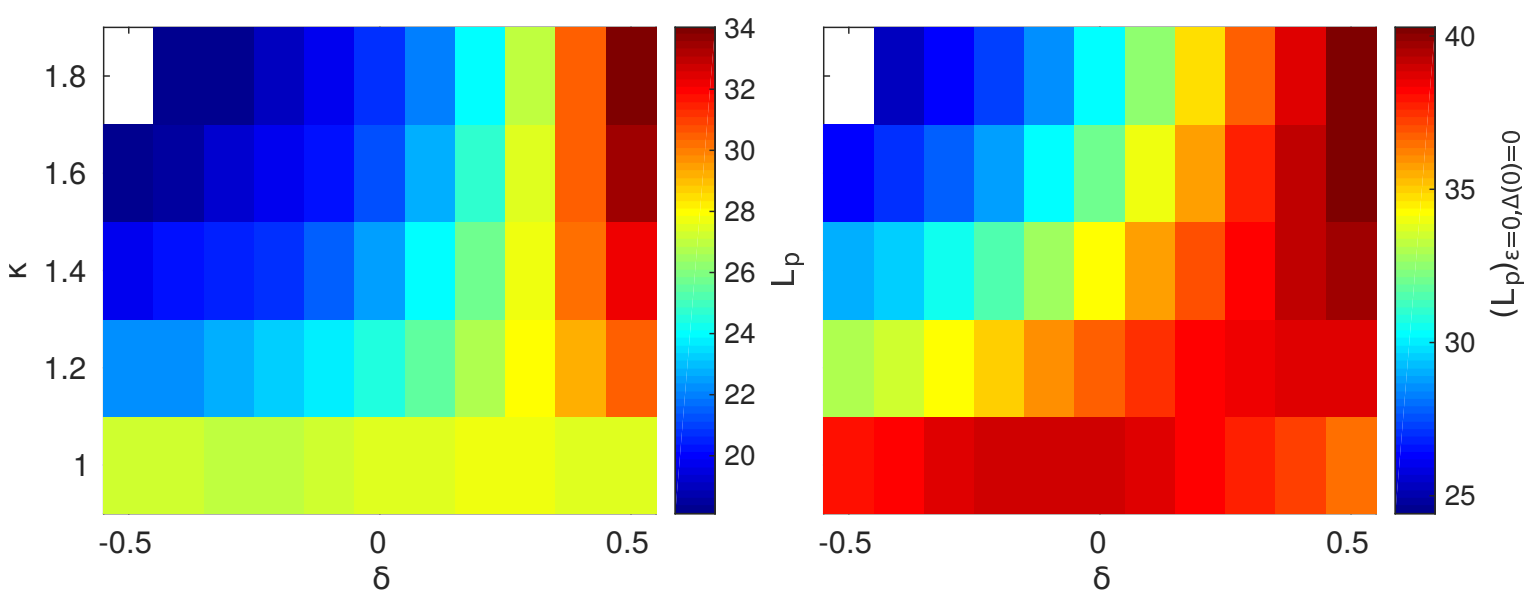

FIG. 4: Value of $L_{p}$ as a function of $\kappa$ and $\delta$ that are solution of Eq. (46). The left panel is obtained for $\epsilon \simeq 0.25$ and $\Delta(0) \simeq 7$, while the right panel is computed for $\epsilon=0$ and $\Delta(0)=0$.

where the mode that dominates turbulent transport is assumed to be the one that maximises the ratio $\gamma / k_{Y}$. We remark that $\gamma$ and $k_{Y}$ depend on $L_{p}$. Therefore, Eq. (46) is an implicit equation in $L_{p}$, and, in general, it must be approached numerically. This is achieved by solving Eqs. (31)-(35) for $\gamma$ over the parameter space $\left(k_{Y}, L_{p}\right)$ with the linear solver detailed in Sec. III, in search for the value of $L_{p}$ that satisfies Eq. (46). Note that in the rest of this section we assume $c_{s}=1$ (i.e. that the reference temperature corresponds to the one at the LCFS) and we consider $m_{i} / m_{e}=2000, \eta=0.66, q=4, R_{0}=500$, and $\hat{s}(a)=0$.

The values of $L_{p}$ that satisfy Eq. (46) for $\epsilon \simeq 0.25$ and $\Delta(0) \simeq 7$ are presented in Fig. 4 (left panel) as a function of $\kappa$ and $\delta$. Several observations can be made based on these results: (i) triangularity has a weak impact on $L_{p}$ when $\kappa=1$; (ii) for $\delta=0, L_{p}$ is reduced by increasing $\kappa$, suggesting that turbulence is suppressed by elongation; (iii) for $\kappa>1, L_{p}$ is reduced for $\delta<0$, indicating that turbulence is suppressed by negative triangularity; and (iv) for $\kappa>1, L_{p}$ is increased for $\delta \gtrsim 0$, the effect becoming more relevant at $\delta \gtrsim 0.3$, meaning that turbulence is enhanced by large positive triangularity.

To isolate the different shaping effects on the SOL width, we perform a $L_{p}$ scan on $\kappa$ and $\delta$ with $\Delta(0)=0$ and $\epsilon=0$. The results are presented in Fig. 4 (right panel). While the same trends previously observed for $\epsilon \simeq 0.25$ and $\Delta(0) \simeq 7$ are recovered, $L_{p}$ is larger, suggesting that Shafranov's shift and $\epsilon$ effects stabilize plasma turbulence. This is in agreement with the observations presented in Sec. III.

Note that the solution of Eq. (46) provides also the $k_{Y}$ value of the mode that leads to most 

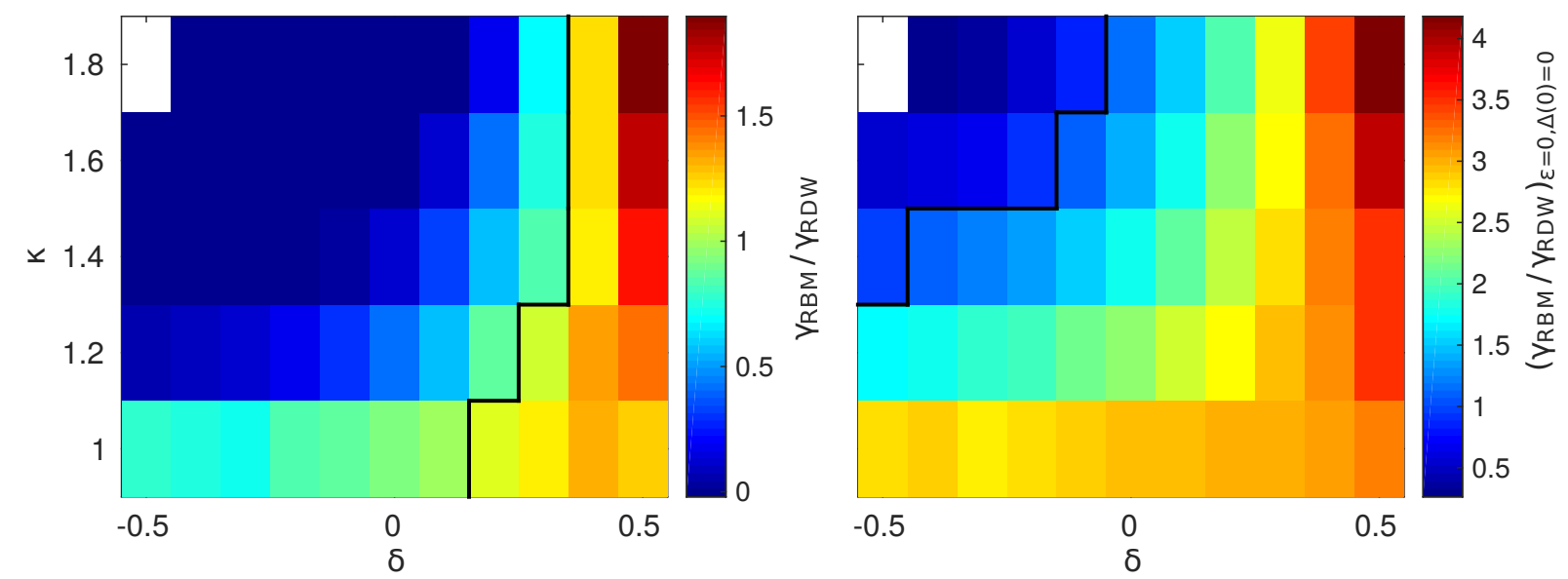

FIG. 5: Ratio between $\gamma_{R B M}$ and $\gamma_{R D W}$ as a function of $\kappa$ and $\delta$, obtained solving Eqs. (37)-(39) and (41)-(44) with the linear code imposing the $L_{p}$ and $k_{Y}$ values that are solution of Eq. (46). The left panel is obtained for $\epsilon \simeq 0.25$ and $\Delta(0) \simeq 7$, while the right panel is computed for $\epsilon=0$ and $\Delta(0)=0$. The black lines indicate the transition between $\gamma_{R D W}>\gamma_{R B M}$ and $\gamma_{R D W}<\gamma_{R B M}$.

of the transport. An analysis of $k_{Y}$ as a function of $\kappa$ and $\delta$ has been performed, showing that plasma shaping has a small impact on its value.

To investigate the impact of the plasma shaping on the turbulent regimes, and therefore to gain a deeper insight on the turbulence properties, we compute the growth rate associated with RBMs $\left(\gamma_{R B M}\right)$ and RDWs $\left(\gamma_{R D W}\right)$ at the $L_{p}$ and $k_{Y}$ solutions of Eq. (46), as a function of $\kappa$ and $\delta$. This is done by solving Eqs. (37)-(39) and (41)-(44) with the linear solver presented in Sec. III. The ratio between $\gamma_{R B M}$ and $\gamma_{R D W}$ as a function of $\kappa$ and $\delta$ for $\epsilon \simeq 0.25$ and $\Delta(0) \simeq 7$ is presented in Fig. 5 (left panel). We observe that: (i) the RBM is the dominant instability for positive values of the triangularity; (ii) the RDW dominates when negative $\delta$ values are considered; (iii) a combination of the two instabilities characterizes the plasma dynamics for $\kappa \simeq 1$ and for $\delta \simeq 0.2-0.3$. This is in agreement with the observations in Sec. III, where it is shown that RDWs are only slightly affected by plasma shaping, while RBMs are strongly stabilized by elongation and negative triangularity.

We also study the impact of Shafranov's shift and $\epsilon$ effects on RBMs and RDWs by performing the same analysis for $\epsilon \simeq 0$ and $\Delta(0)=0$. The results (see Fig. 5, right panel) show that Shafranov's shift and $\epsilon$ effects stabilize the RBMs, leading to a decrease of the equilibrium pressure gradient length, as discussed earlier.

We now deduce an analytical scaling of $L_{p}$ as a function of $\kappa$ and $\delta$. Since RBMs are 
strongly affected by plasma shaping, while the $\left(\gamma / k_{Y}\right)_{\max }$ ratio associated with RDWs depends weakly on the magnetic shape [variation of $\left(\gamma / k_{Y}\right)_{\max }$ less than $30 \%$ for $1 \leq \kappa \leq 1.8$ and $-0.5 \leq \delta \leq 0.5]$, we derive the analytical scaling of $L_{p}$ considering the RBMs dispersion relation only, and we assume that the resulting scaling breaks down if $L_{p, a}<L_{p, R D W}$, where $L_{p, a}$ is the result of the RBM analytical scaling and $L_{p, R D W}$ is the equilibrium pressure gradient length associated with RDWs derived from Eqs. (45) and (46) for a circular magnetic geometry $\left(L_{p, R D W}=27.7\right.$ for $\nu=0.1, q=4, R_{0}=500$, and $\left.\eta=0.66\right)$.

Having observed that magnetic shape affects the RBM growth rate mostly through the curvature operator, we can assume a strongly ballooned mode around $\theta_{*}=0, k_{Z} \sim 1 / q$, and $\mathcal{C}^{Y} \simeq-\left.\partial_{r} R(r, \theta)\right|_{\theta_{*}=0}$ to simplify Eqs. (37)-(39). Moreover, imposing $\epsilon=0$ and $\Delta(0)=0$ for the sake of simplicity, we evaluate $\partial_{k_{Y}}\left(\gamma / k_{Y}\right)=0$ to identify the largest $\gamma / k_{Y}$ ratio, obtaining

$$
\gamma^{2}=\gamma_{b}^{2} \frac{C(\kappa, \delta, q)}{3}, \quad k_{Y}^{2}=\sqrt{3} \frac{k_{b}^{2}}{2} C(\kappa, \delta, q)^{-1 / 2}
$$

where

$$
C(\kappa, \delta, q)=\left.\partial_{r} R_{c}(r, \theta)\right|_{\theta_{*}=0}=1+\frac{\delta q}{1+q}+\frac{\delta^{2}(7 q-1)}{16(1+q)}-\frac{\kappa-1}{2(\kappa+1)}-\frac{(\kappa-1)(5 q-2)}{(\kappa+1)^{2}(2+q)},
$$

as derived in Appendix C. Plugging these values into Eq. (46), we derive

$$
L_{p, a}=\frac{2^{5 / 7}}{3^{3 / 7}} C(\kappa, \delta, q)^{3 / 7} \nu^{2 / 7} q^{8 / 7} R_{0}^{3 / 7}
$$

Equation (49) is a generalization of the scaling derived in Refs. [20, 21, 31] to include noncircular magnetic geometries and allows us to predict the SOL width of inner-wall limited discharges from first-principle arguments when elongation and non-zero triangularity are considered. In Fig. 6 we present the result of this scaling. We recover the same trends observed in Fig. 4 and the transition between RDWs and RBMs, taking place at $L_{p, a}=$ $L_{p, R D W}$ and indicated by the black line, in good agreement with the condition $\gamma_{R B M} / \gamma_{R D W}=$ 1 of Fig. 5 (right panel). However, we notice two main differences between the analytical scaling and the numerical results: (i) for $\kappa=1$ the analytical scaling shows that $L_{p}$ depends on $\delta$ while $L_{p}$ is almost independent of $\delta$ in Fig. 4 ; and (ii) for $\delta>0.3$ the value of $L_{p}$ decreases by increasing the plasma elongation, while the opposite behavior is observed in the results of Fig. 4. By solving Eq. (46) within each assumption made in the derivation of Eq. (49), we observe that the differences between the analytical scaling and the numerical 


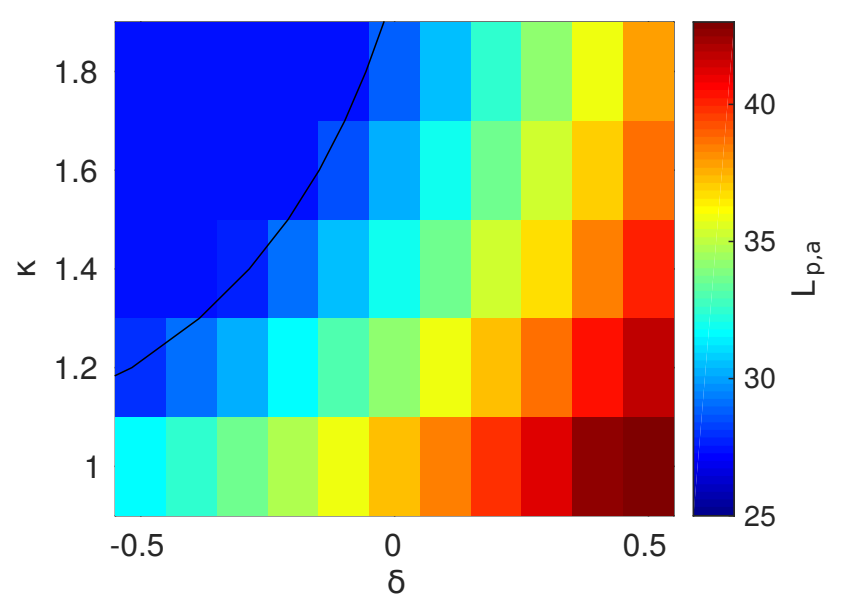

FIG. 6: Value of $L_{p}$ as a function of $\kappa$ and $\delta$, obtained from Eq. (49) for $q=4, \nu=0.1$, and $R_{0}=500$. The black line indicates $L_{p}(\kappa, \delta)=L_{p, R D W}$. Note that in the top-left corner we impose $L_{p}=L_{p, R D W}$.

results are mainly due to the approximation $\left.\mathcal{C}^{Y} \approx \mathcal{C}^{Y}\right|_{\theta_{*}=0}$. In fact, the global magnetic geometry has an effect on the RBM that cannot be correctly captured by the modification of the curvature at the outer midplane.

\section{NON-LINEAR SIMULATIONS}

In this section we carry out a set of non-linear simulations of the SOL plasma dynamics using the GBS code [15] and compare these with the results presented in Secs. III and IV. We first describe the numerical scheme implemented in GBS, focusing on the modifications introduced to generalize the magnetic geometry of the code. We then present the non-linear simulation results and their comparison with our theoretical findings.

\section{A. Implementation and numerics}

The model presented in Sec. II is now implemented in the GBS code [15]. GBS was developed in the last few years to simulate the open field line region of magnetic confinement devices, evolving the drift-reduced Braginskii equations presented in Sec. II A without any separation between plasma background and fluctuations. The development of GBS was carried out by considering increasingly complex magnetic geometries. First, the code was used to simulate linear devices such as LAPD, and simple magnetized toroidal devices, such as 
TORPEX [47, 59-61]. The code was then extended to include tokamak geometries [15], and it is now used to model the tokamak SOL for limited plasmas in arbitrary magnetic geometry. The numerical scheme implemented in GBS was subject to a rigorous verification, and the simulation results were validated against several experimental measurements [36, 37, 39, 62]. A detailed description of the code can be found in Ref. [15].

For completeness, we present here a brief summary of the main properties of GBS. Equations (1)-(5) and Eqs. (6)-(11) are expressed using the toric coordinate system $(y, x, z)$ described in Sec. II B, and are integrated in time with a fourth order Runge-Kutta algorithm. Spatial derivatives on the right-hand side of Eqs. (1)-(5) are discretized using second order finite difference schemes, except for the Poisson's brackets, which are evaluated using the Arakawa scheme [63]. In order to take advantage of the strong anisotropy of SOL turbulence, the grid is aligned with the magnetic field and the parallel gradient is computed along the field line. The magnetic geometry is treated with the approximation of being radially local, i.e. geometric coefficients are assumed not to depend on $x$ in the simulated domain. This approximation corresponds to neglecting the magnetic shear effects and assume that $L_{p} \ll a$, which is a reasonable hypothesis, as proven a posteriori by the results presented in the next section.

Note that the computation of the $\nabla_{\perp}^{2}$ operator is not straightforward. As a matter of fact, the discretization of the operator $\nabla_{\perp}^{2}$ can introduce numerical instabilities with positive growth rate if $\nabla_{\perp}^{2}$ is represented with a non-symmetric real matrix $\mathbf{D}$. Therefore, to ensure the self-adjointness of $\mathbf{D}$, we write

$$
\nabla_{\perp}^{2} A=\frac{\partial}{\partial x}\left(\mathcal{N}^{x x} \frac{\partial A}{\partial x}+\frac{\mathcal{N}^{x y}}{2} \frac{\partial A}{\partial y}\right)+\frac{\partial}{\partial y}\left(\mathcal{N}^{y y} \frac{\partial A}{\partial y}+\frac{\mathcal{N}^{x y}}{2} \frac{\partial A}{\partial x}\right)+\mathcal{N}^{\prime x} \frac{\partial A}{\partial x}+\mathcal{N}^{\prime y} \frac{\partial A}{\partial y}
$$

with $\mathcal{N}^{\prime x}=\mathcal{N}^{x}-\partial_{x} \mathcal{N}^{x x}-\partial_{y} \mathcal{N}^{x y} / 2$ and $\mathcal{N}^{\prime y}=\mathcal{N}^{y}-\partial_{y} \mathcal{N}^{y y}-\partial_{x} \mathcal{N}^{x y} / 2$, and we neglect the $\mathcal{N}^{\prime x}$ and $\mathcal{N}^{\prime y}$ terms with respect to the $\mathcal{N}^{x x}, \mathcal{N}^{x y}$, and $\mathcal{N}^{y y}$ terms, since the first two terms are usually $a$ times smaller than the last three, and they are therefore expected to have a negligible impact on the simulation results.

Finally, we note that the geometric coefficients in Eqs. (18)-(20) are computed with the same numerical scheme presented in Sec. III and used for the coefficients in Eqs. (21)(24). Also, we introduce the aspect ratio effects entering the various magnetic coefficients through a parameter $\epsilon$ that is varied separately from the considered domain, i.e. we allow for $\epsilon R_{0} \neq L_{y} /(2 \pi)$ when evaluating the coefficients of Eq. (18). However, we note that, for 
the simulations with $\epsilon \simeq 0.25$, the equality $\epsilon R_{0}=L_{y} /(2 \pi)$ is satisfied.

\section{B. Simulation results}

We use GBS to carry out eight non-linear simulations, with $(\kappa, \delta)=$ $(1,0),(1.8,0),(1.8,-0.3),(1.8,0.3)$, considering in one case $\epsilon \simeq 0.25$ and $\Delta(0) \simeq 7$, and in the other $\epsilon=0$ and $\Delta(0)=0$. Other relevant physical parameters are $q=4, \nu=0.1$, $R_{0}=500, m_{i} / m_{e}=200, L_{y}=800$, and $L_{x}=150$, being $L_{x}$ the radial size of the domain, extending from $x_{i}$ to $x_{o}$, with $x_{i}=a-50$ and $x_{o}=a+100$. The particle and electron temperature sources, used to mimic the plasma outflow from the core, are modeled as $S_{n, T_{e}}=\exp \left[-(x-a)^{2} / \sigma^{2}\right]$, with $\sigma=2.5$. Since most of the particles coming from the core are lost at the limiter plates, preventing them from reaching the vessel wall, the conditions applied at $x=x_{o}$, the outer edge of the simulation domain, should not significantly impact turbulence. Therefore, a buffer region is located between $x=a+90$ and $x=x_{o}$, and $a d$ $h o c$ boundary conditions (Dirichelet for $\phi$ and $\omega$, and Neumann for $n, v_{\| i}, v_{\| e}$, and $T_{e}$ ) are applied at $x=x_{o}$. On the other hand, at the LCFS, the plasma outflow from the core is mimicked by the source terms. These sources are located at a distance of 50 units from the inner boundary of the computational domain, and the domain between $x=x_{i}$ and $x=a$ is used as buffer region and it is not taken into account for turbulence analysis. Consequently, also at $x=x_{i}$, ad hoc boundary conditions (the same applied at $x_{o}$ ) are used, and we verified that their impact on turbulence properties is not significant.

As $\gamma m_{e} / m_{i}<\nu$, we expect that the unphysical value of the mass ratio does not influence the results [48]. The simulations are carried out with the following numerical parameters: $N_{x}=192, N_{y}=512, N_{z}=64, \eta_{i} \approx \eta_{e} \approx 2, \chi_{\|, T_{e}}=0.2$, and all the perpendicular diffusion coefficients are in the range $5-10$. We remark that the value of the dissipative coefficients does not affect significantly the simulation results (simulations carried out with $\chi_{\|, T_{e}}=100$ show a $\sim 20 \%$ steeper $L_{p}$.) In Fig. 7 we present typical poloidal snapshots of the plasma pressure for $\epsilon=0$ and $\Delta(0)=0$. One observes the presence of turbulent eddies that transport plasma radially outward. In the two simulations with $(\kappa, \delta)=(1.8,0),(1.8,-0.3)$ plasma turbulence penetrates considerably less in the SOL with respect to a circular magnetic geometry. This is consistent with Sec. IV results, which show that elongation and negative triangularity decrease $L_{p}$. In these two simulations, turbulence results to be 

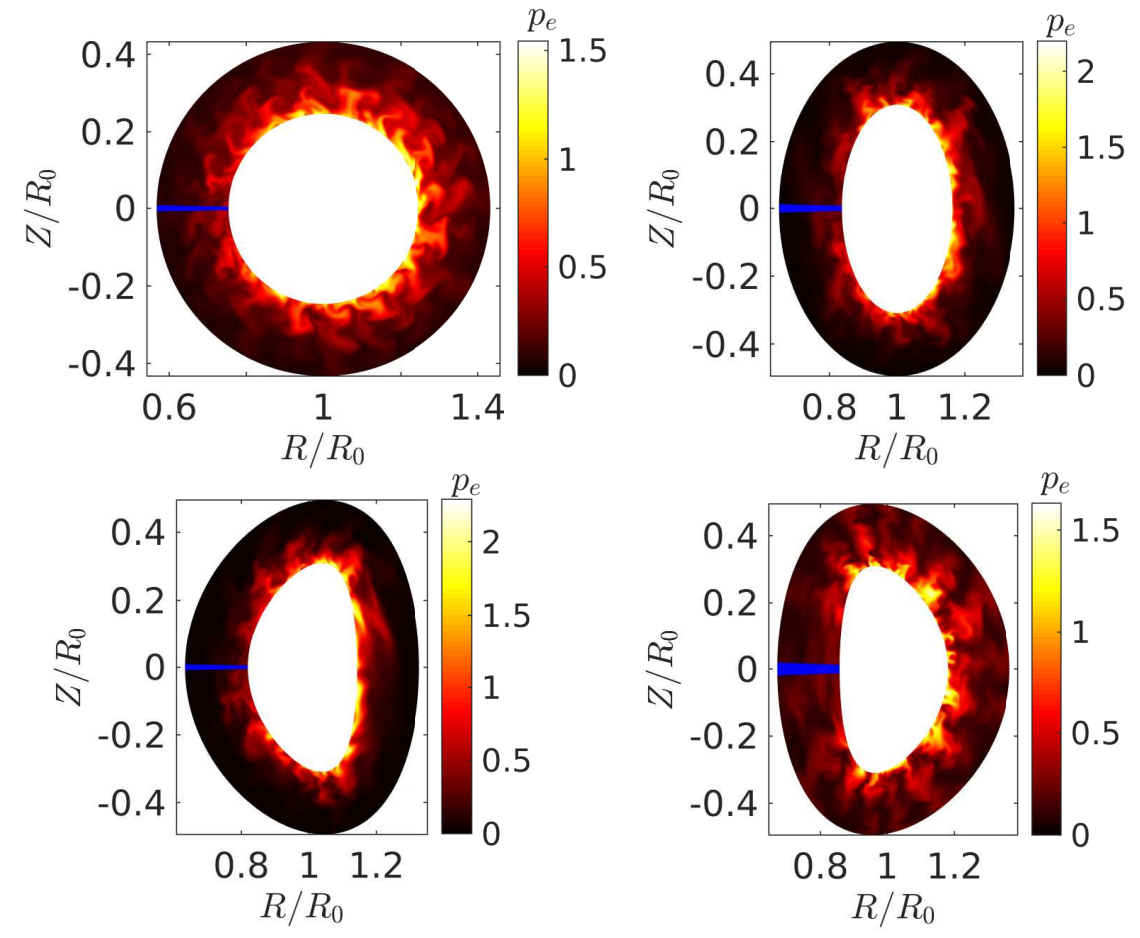

FIG. 7: Poloidal cross sections of the electron pressure plasma profile resulting from non-linear simulations carried out for $\epsilon=0$ and $\Delta(0)=0$, extending from $x=a$ to $x=a+90$. The four magnetic geometries considered are characterized by $\kappa=1$ and $\delta=0$ (top left panel), $\kappa=1.8$ and $\delta=0$ (top right panel), $\kappa=1.8$ and $\delta=-0.3$ (bottom left panel), and $\kappa=1.8$ and $\delta=0.3$ (bottom right panel). The limiter is indicated by a blue line at the inner midplane.

suppressed in particular at the outer midplane, in agreement with the findings in Sec. III, which shows that RBMs are stabilized by elongation and negative triangularity. On the other hand, plasma turbulence appears to have similar amplitude in a circular magnetic geometry and for $\kappa=1.8$ and $\delta=0.3$, in agreement with Sec. IV results.

We note that the four simulations carried out with $\epsilon \simeq 0.25$ and $\Delta(0) \simeq 7$ (see Fig. 8) display the same trends, but their differences are less pronounced than in the $\epsilon=0$ and $\Delta(0)=0$ case. Therefore, in the following we focus our attention on the four simulations with $\epsilon=0$ and $\Delta(0)=0$, where the differences are larger, and we briefly discuss the results of the simulations with $\epsilon \simeq 0.25$ and $\Delta(0) \simeq 7$.

To investigate the nature of the turbulence present in the non-linear simulations, recall that the phase shift between plasma potential and pressure fluctuations is close to 0 when DWs drive the plasma dynamics, while it is close to $\pi / 2$ for BM dominated turbulence. 

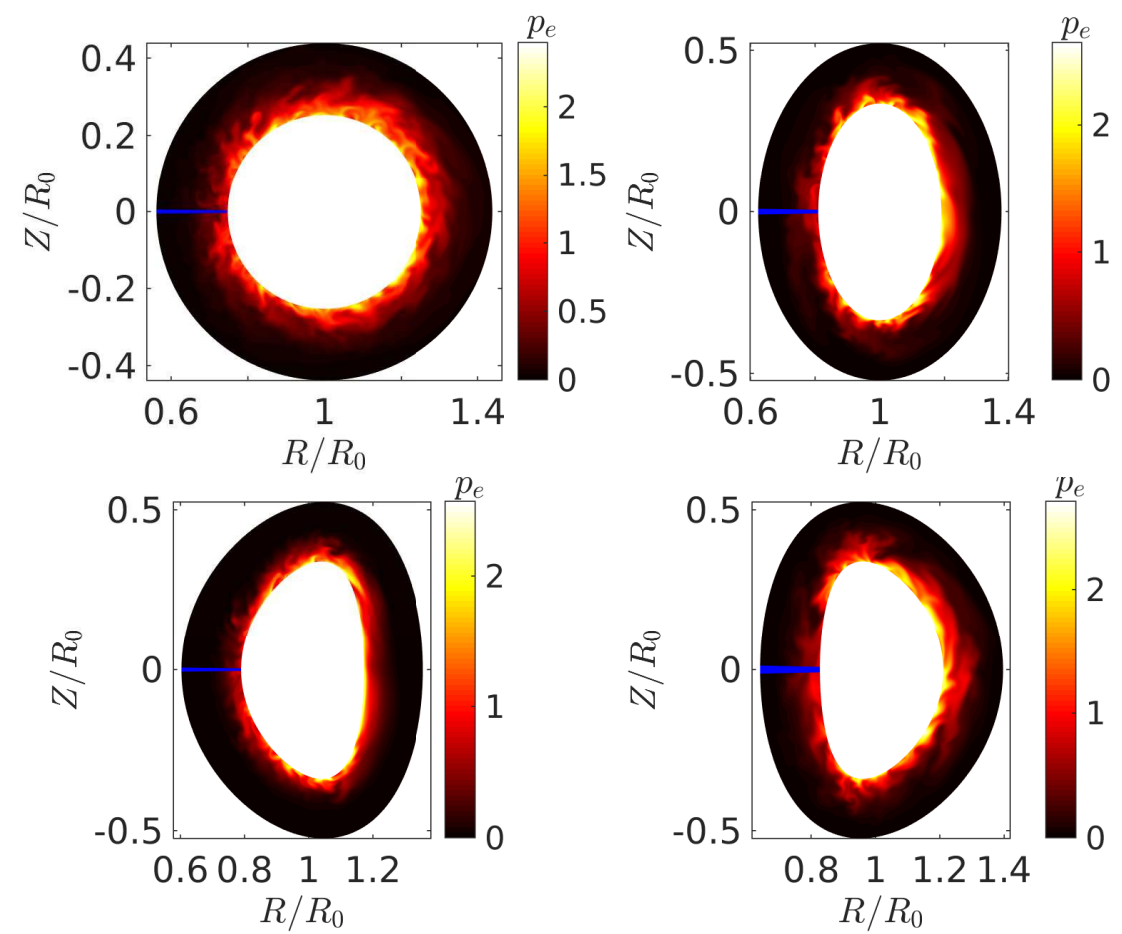

FIG. 8: Poloidal cross sections of the electron pressure plasma profile resulting from non-linear simulations carried out for $\epsilon \simeq 0.25$ and $\Delta(0) \simeq 7$, extending from $x=a$ to $x=a+90$. The four magnetic geometries considered are characterized by $\kappa=1$ and $\delta=0$ (top left panel), $\kappa=1.8$ and $\delta=0$ (top right panel), $\kappa=1.8$ and $\delta=-0.3$ (bottom left panel), and $\kappa=1.8$ and $\delta=0.3$ (bottom right panel). The limiter is indicated by a blue line at the inner midplane.

Moreover, since electrons are close to adiabaticity for DWs, electron pressure and potential fluctuations are correlated, while they are not for BMs [64]. Therefore, following the procedure suggested in Ref. [48], we compute the probability distribution function of the phase shift between the electron pressure and the potential fluctuations and the joint probability of the electron pressure and the potential fluctuations normalized to their standard deviation for $\theta_{*} \in[-\pi, \pi]$ and at $x-a=L_{p} \ln 2$, over a time interval of about 30 units, for each of the four simulations with $\epsilon=0$ and $\Delta(0)=0$, and we present the results in Figs. 9 and 10. In the simulations with $(\kappa, \delta)=(1,0),(1.8,0.3)$ the phase shift is closer to $\pi / 2$ and electron pressure and potential fluctuations are less correlated, with a correlation coefficient of 0.66 and 0.73 , respectively, indicating that RBMs contribute to the SOL dynamics. On the other hand, for $\kappa=1.8$ and $\delta=-0.3$, the phase shift is close to 0 and the electron pressure and potential fluctuations are more correlated, with a correlation 


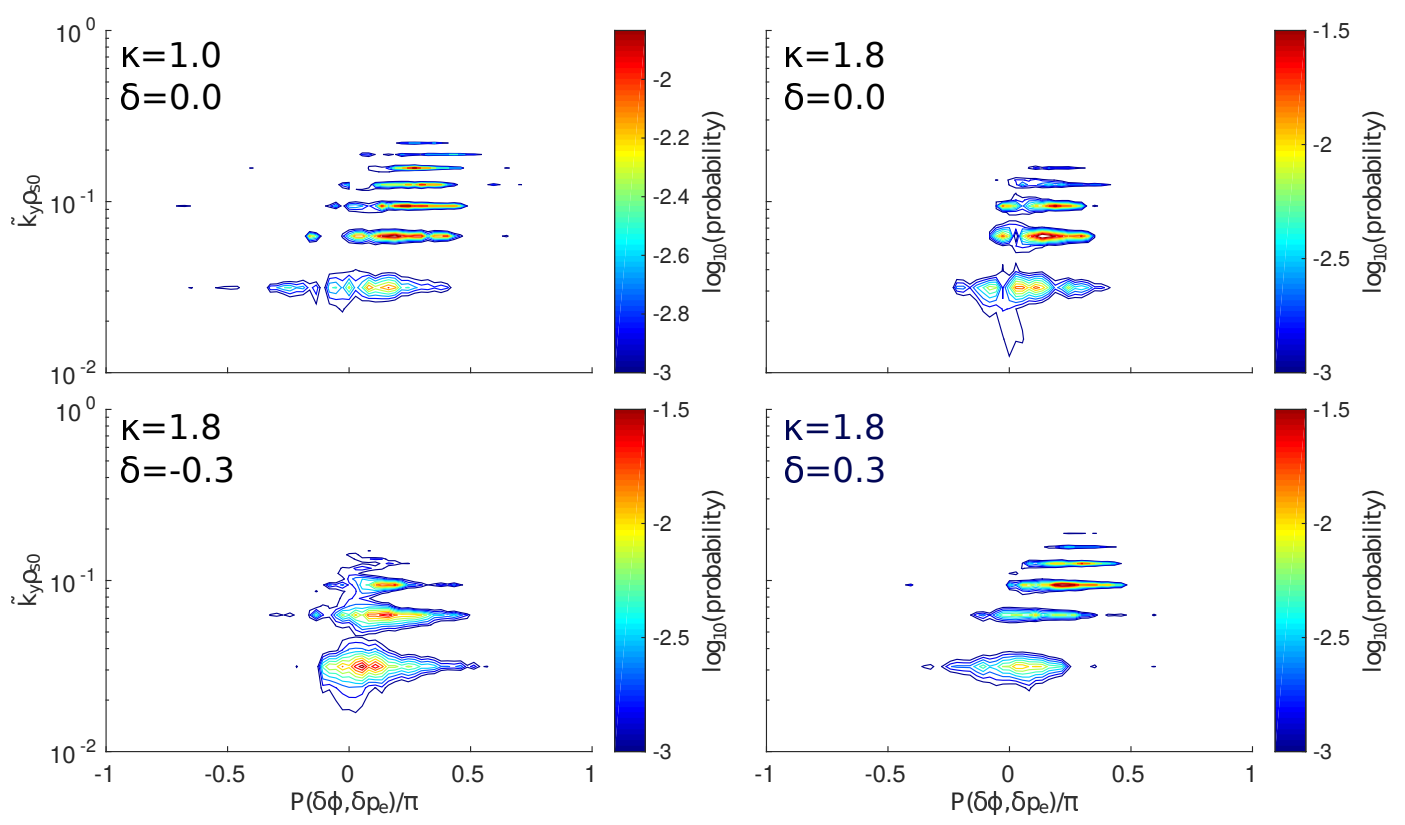

FIG. 9: Probability distribution function of the phase shift between the electron pressure and the potential fluctuations resulting from non-linear simulations carried out for $\epsilon=0$ and $\Delta(0)=0$. The four magnetic geometries considered are characterized by $\kappa=1$ and $\delta=0$ (top left panel), $\kappa=1.8$ and $\delta=0$ (top right panel), $\kappa=1.8$ and $\delta=-0.3$ (bottom left panel), and $\kappa=1.8$ and $\delta=0.3$ (bottom right panel).

coefficient of 0.84 , suggesting that RDWs is the turbulence drive. For $\kappa=1.8$ and $\delta=0$ it is not possible to clearly discriminate between RBMs and RDWs, since the electron pressure and potential fluctuations have a correlation coefficient of 0.82 , similar to the simulation preformed for $\kappa=1.8$ and $\delta=-0.3$, but the phase shift is larger than 0 . We note that, performing the same analysis for the four simulations with $\epsilon \simeq 0.25$ and $\Delta(0) \simeq 7$, we find that RDWs drive turbulence except for $\kappa=1$ and $\delta=0$, where a combination of RBMs and RDWs is responsible of the turbulent dynamics. These results are in agreement with Secs. III and IV findings.

Finally, to perform a quantitative comparison between the estimate of $L_{p}$ given by Eq. (46), the analytical scaling in Eq. (49), and the non-linear simulation results, we time-average the pressure profiles provided by GBS over an interval of about 30 time units, and over four time subdomains, each of about 7.5 time units. The results are then averaged over the toroidal angle and the averages fitted at the outer midplane with $p_{e}(x)=p_{e, a} \exp \left[-(x-a) / L_{p}\right]$. The fit over the interval of 30 time units provides $L_{p}$, while the difference between the 

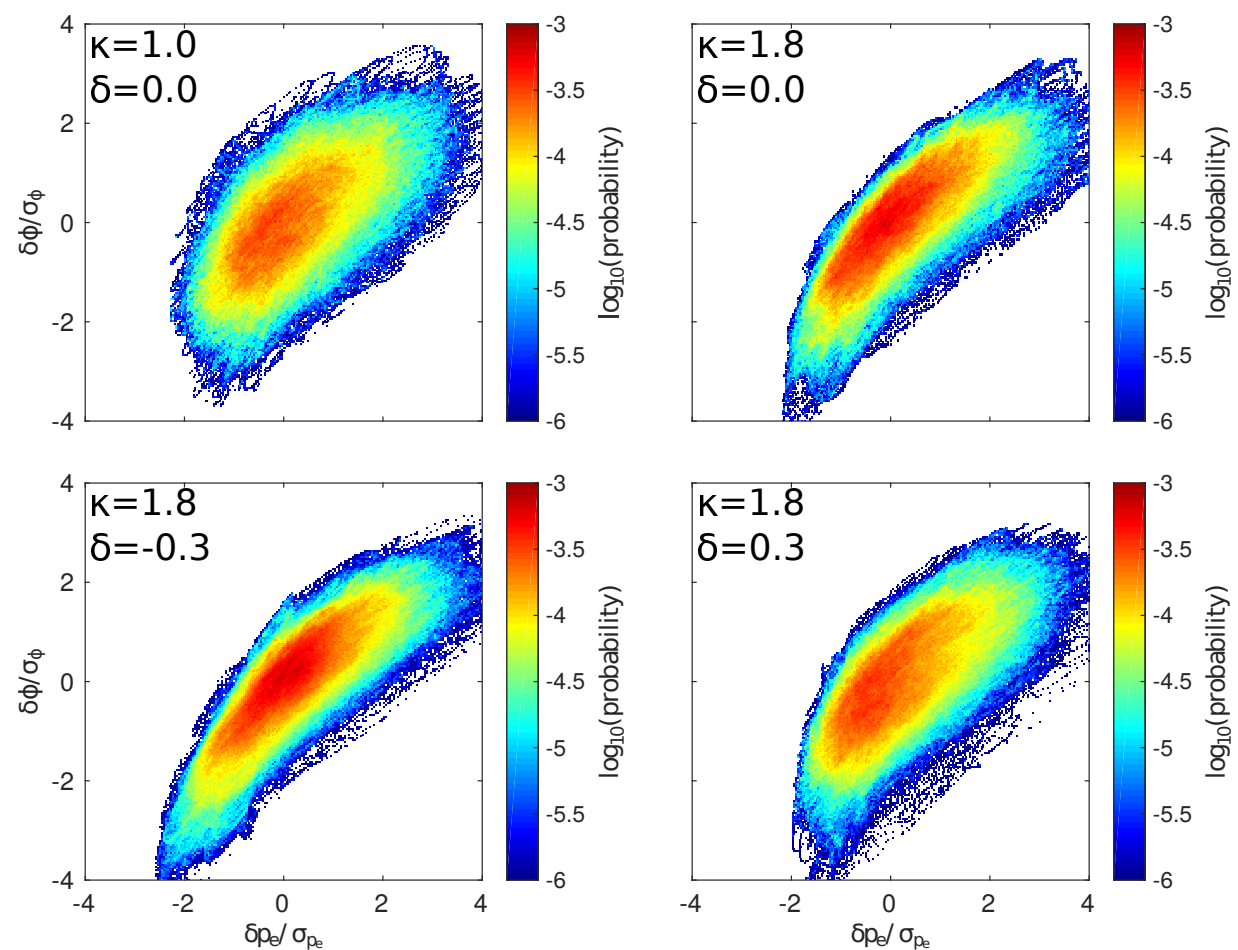

FIG. 10: Joint probability of the electron pressure and the potential fluctuations normalized to their standard deviation resulting from non-linear simulations carried out for $\epsilon=0$ and $\Delta(0)=0$. The four magnetic geometries considered are characterized by $\kappa=1$ and $\delta=0$ (top left panel), $\kappa=1.8$ and $\delta=0$ (top right panel), $\kappa=1.8$ and $\delta=-0.3$ (bottom left panel), and $\kappa=1.8$ and $\delta=0.3$ (bottom right panel).

\begin{tabular}{cccccc}
\hline \hline \multirow{2}{*}{$\kappa, \delta)$} & Non - linear sim. & Eq. (46) & Non - linear sim. & Eq. (46) & Eq. (49) \\
& $\epsilon \simeq 0.25, \Delta(0) \simeq 7$ & $\epsilon \simeq 0.25, \Delta(0) \simeq 7$ & $\epsilon=0, \Delta(0)=0$ & $\epsilon=0, \Delta(0)=0$ & \\
\hline$(1.0,0.0)$ & $25 \pm 1$ & 27.4 & $37 \pm 2$ & 38.9 & 37.1 \\
$(1.8,0.0)$ & $20 \pm 1$ & 20.7 & $26 \pm 3$ & 30.3 & 28.8 \\
$(1.8,-0.3)$ & $15 \pm 1$ & 18.1 & $20 \pm 1$ & 26.2 & 27.7 \\
$(1.8,0.3)$ & $23 \pm 1$ & 26.8 & $43 \pm 3$ & 36.8 & 34.1 \\
\hline
\end{tabular}

TABLE I: Values of $L_{p}$ obtained from the non-linear simulations, from Eq. (46), and from Eq. (49).

$L_{p}$ s obtained over the four subdomains gives an estimate of its uncertainty. Applying this methodology, and computing the $L_{p}$ given by Eq. (46) and Eq. (49), we obtain the results listed in Table. I. Note that, since Eq. (49) gives $L_{p}=23.4$ for $\kappa=1.8$ and $\delta=-0.3$, we replace this value with $L_{p, R D W}$ in Table I. Several observations can be made based on 
these results: (i) Eq. (46) is in fairly good agreement with the results obtained from the non-linear simulations, indicating that the gradient removal theory is able to predict $L_{p}$ even when non-circular magnetic geometries are considered; and (ii) if $\epsilon=0$ and $\Delta(0)=0$, Eq. (49) provides an estimate of $L_{p}$ that is in good agreement with Eq. (46), meaning that the analytical scaling correctly describes the dependence of $L_{p}$ on $\kappa$ and $\delta$. We remark that the results obtained in Table I for the non-linear simulations are obtained at the outer midplane. However, we note that similar results are obtained also when considering the poloidally averaged plasma pressure profile.

\section{DISCUSSION}

In the previous sections it has been discussed the impact of plasma shaping on SOL turbulence, revealing that elongation, triangularity, Shafranov's shift, and finite aspect ratio effects strongly impact the growth rate of RBMs. To intuitively explain this result, we represent in Fig. 11 the magnetic field lines that characterize the four simulations with $\epsilon=0$ and $\Delta(0)=0$ in the $\varphi-\theta$ plane and at fixed radial position $x=a$. We observe that for the two simulations with $(\kappa, \delta)=(1,0),(1.8,0.3)$, magnetic field lines are almost straight and their slope is close to $1 / q=0.25$ at the outer midplane. On the other hand, the two simulations with $(\kappa, \delta)=(1.8,0),(1.8,-0.3)$ are characterized by magnetic field lines that are stretched in the poloidal direction and their slope strongly increases close to $\theta=0$. A detailed investigation of the impact of $\kappa$ and $\delta$ on the magnetic field lines indicates that values of $\kappa>1$ and $\delta<0$ stretch the magnetic field lines in the poloidal direction near $\theta=0$, while they are compressed for large positive triangularities. This suggest that particles trajectories lies longer in the bad curvature region in a circular magnetic geometry, or for $\delta>0$, with respect to the case of $\kappa>1$ or $\delta<0$. Since BMs are strongly destabilized at the outer midplane, i.e. in the proximity of $\theta=0$, elongation and negative triangularity result in a stabilization of BMs, as observed in the simulation results previously discussed. The same argument can be used to explain the impact of $\Delta$ and $\epsilon$. As a matter of fact, magnetic field lines are even more stretched in the proximity of $\theta=0$ when Shafranov's shift and finite aspect ratio effects are included. We remark that in Sec. II C we computed the Shafranov's shift neglecting the plasma pressure contribution. Since $\Delta(0)$ usually increases almost linearly with $\beta_{p}$, we expect that including the plasma pressure contribution in the 

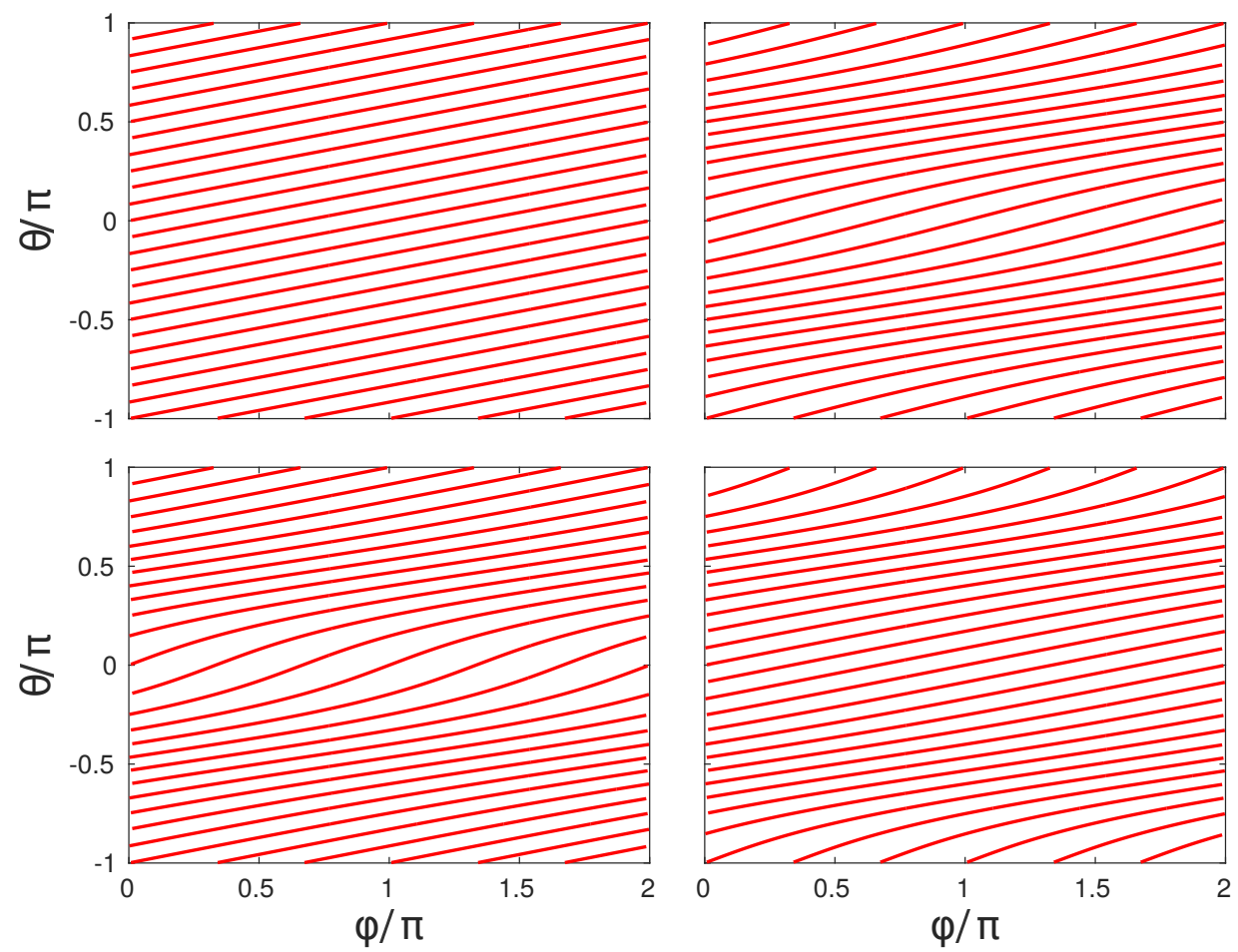

FIG. 11: Magnetic field lines in the $\varphi-\theta$ plane, at $x=a$, for $\epsilon=0$ and $\Delta(0)=0$. The four magnetic geometries considered are characterized by $\kappa=1$ and $\delta=0$ (top left panel), $\kappa=1.8$ and $\delta=0$ (top right panel), $\kappa=1.8$ and $\delta=-0.3$ (bottom left panel), and $\kappa=1.8$ and $\delta=0.3$ (bottom right panel).

magnetic equilibrium stabilizes the RBMs and decreases $L_{p}$.

Finally, we note that our findings are in agreement with the experimental observations of the impact of plasma shaping on the SOL width, which show that $L_{p}$ decreases with $\kappa$ [27]. Moreover, we would like to point out that plasma shaping seems to have a similar impact both on core and SOL turbulence. In fact, Refs. [22-25] pointed out that core turbulence is (i) usually stabilised by elongation, except for large positive triangularities, for which it is enhanced, (ii) stabilised by negative triangularity, and (iii) destabilised by large positive triangularity.

\section{CONCLUSIONS}

In the present paper, the effects of plasma shaping on SOL turbulence are discussed. Depending on the magnetic geometry, the SOL turbulence regime is identified, and the impact 
of Shafranov's shift, finite aspect ratio, elongation, and triangularity on $L_{p}$ is investigated. The results obtained from the linear theory are compared with non-linear simulations.

The drift-reduced Braginskii equations are derived for arbitrary magnetic geometries and, using the flux-tube and the toric coordinate systems, the coefficients characterizing the differential operators entering the model equations are computed. Moreover, the analytical model used to express the dependence of the magnetic equilibrium on $\epsilon, \Delta, \kappa$, and $\delta$ is presented. This model allows isolating the different shaping effects that affect SOL turbulence and to investigate their impact on SOL instabilities.

First, the influence of the plasma shaping on the two main instabilities driving the SOL turbulence, the RBMs and the RDWs, and the effect of $\epsilon, \Delta, \kappa$, and $\delta$ on their growth rate are discussed. It turns out that plasma shaping strongly impacts the RBM's growth rate, while RDWs are considerably less affected. In particular, it is observed that $\epsilon, \Delta$, $\kappa$, and $\delta<0$ effects stabilize RBMs, while these are enhanced for $\delta>0$.

Second, a non-linear saturation theory of the growth of the unstable linear modes is presented, and it is used to estimate the SOL width from a linear analysis of the main instability driving the SOL turbulence. It is found that $L_{p}$ decreases for $\kappa>1$ and $\delta<0$, while it increases for $\delta>0.2$. This result is used to identify the turbulent regime depending on the shaping parameters. It turns out that, for $\kappa>1$ and $\delta<0$, RDWs drive the SOL dynamics, while RBMs dominate for large positive $\delta$ values, or if $\kappa=1$ and aspect ratio effects are neglected. Moreover, assuming that RBMs are mainly affected by plasma shaping through the curvature operator, an analytical scaling for $L_{p}$ is derived, taking into account $\kappa$ and $\delta$ effects. This analytical scaling generalizes the scaling presented in Refs. [20, 21, 31] by including non-circular magnetic geometries.

Third, the results obtained from the linear theory are compared with non-linear simulations carried out with the flux-driven fluid code GBS, showing good qualitative and quantitative agreement, and, finally, an intuitive explanation for the strong impact of $\kappa$ and $\delta$ on the RBMs growth rate is given, showing that elongation and negative triangularity result in a stabilization of RBMs. 


\section{Acknowledgments}

The authors gratefully acknowledge useful discussions with J. P. Graves, J. Loizu, and P. Molina Cabrera. The simulations presented herein were carried out in part using the HELIOS supercomputer system at Computational Simulation Centre of International Fusion Energy Research Center (IFERC-CSC), Aomori, Japan, under the Broader Approach collaboration between Euratom and Japan, implemented by Fusion for Energy and JAEA; and in part at the Swiss National Supercomputing Center (CSCS) under Project ID s549. This work has been carried out within the framework of the EUROfusion Consortium and has received funding from the Euratom research and training programme 2014-2018 under grant agreement No 633053. The views and opinions expressed herein do not necessarily reflect those of the European Commission.

\section{APPENDIX A: DERIVATION OF THE OPERATOR COEFFICIENTS IN GEN-} ERAL GEOMETRY

To obtain the operator coefficients presented in Sec. II B, we proceed as follows. First, we define the covariant basis $\nabla \xi^{i}$, being $\left\{\xi^{i}\right\}=\left\{\xi^{1}, \xi^{2}, \xi^{3}\right\}$ an arbitrary set of coordinates, and we write $\mathbf{B}=B_{i} \nabla \xi^{i}$, where $B_{i}$ are the covariant components of $\mathbf{B}$ and the Einstein's summation convention is employed. Note that the contravariant components of the vector $\mathbf{B}$ are evaluated as $B^{i}=\mathbf{B} \cdot \nabla \xi^{i}$, the covariant components as $B_{i}=g_{i j} B^{j}$, the contravariant components of the metric tensor associated with the covariant basis $\nabla \xi^{i}$ as $g^{i j}=\nabla \xi^{i} \cdot \nabla \xi^{j}$, and the covariant metric tensor is defined as the inverse of the contravariant metric tensor. The Jacobian associated with the coordinate transformation $\left\{\xi^{i}\right\}$ is $\mathcal{J}_{\xi^{1}} \xi^{2} \xi^{3}=1 / \sqrt{\operatorname{det}\left(g^{i j}\right)}$, where $\operatorname{det}\left(g^{i j}\right)$ is the determinant of the contravariant metric tensor $g^{i j}$.

Then, to compute the contravariant metric tensor $g^{i j}$ of the toroic and flux tube coordinate systems, we introduce the standard cylindrical coordinate system $\left(R_{c}, \varphi, Z_{c}\right)$, with the $Z_{c}$ axis coinciding with the tokamak symmetry axis and $R_{c}$ the distance from this axis. Assuming axisymmetry, that is $R_{c}=R_{c}(r, \theta)$ and $Z_{c}=Z_{c}(r, \theta)$, the components of the covariant metric 
tensor associated with the coordinate system $(\theta, r, \varphi)$ are defined as

$$
\begin{aligned}
g_{\theta \theta} & =\left(\frac{\partial R_{c}}{\partial \theta}\right)^{2}+\left(\frac{\partial Z_{c}}{\partial \theta}\right)^{2} \\
g_{\theta r} & =\frac{\partial R_{c}}{\partial r} \frac{\partial R_{c}}{\partial \theta}+\frac{\partial Z_{c}}{\partial r} \frac{\partial Z_{c}}{\partial \theta} \\
g_{r r} & =\left(\frac{\partial R_{c}}{\partial r}\right)^{2}+\left(\frac{\partial Z_{c}}{\partial r}\right)^{2} \\
g_{\varphi \varphi} & =R_{c}^{2} \\
g_{r \varphi} & =g_{\theta \varphi}=0 .
\end{aligned}
$$

These expressions allow us to express the contravariant metric tensor components associated with the toric and flux-tube coordinate systems, which are

$$
\begin{aligned}
g^{\theta_{*} \theta_{*}} & =\left(\frac{\partial \theta_{*}}{\partial \theta}\right) g^{\theta \theta}+2 \frac{\partial \theta_{*}}{\partial \theta} \frac{\partial \theta_{*}}{\partial \theta} g^{\theta r}+\left(\frac{\partial \theta_{*}}{\partial r}\right)^{2} g^{r r} \\
g^{\theta_{*} r} & =\frac{\partial \theta_{*}}{\partial r} g^{r r}+\frac{\partial \theta_{*}}{\partial \theta} g^{\theta r} \\
g^{\theta_{*} r} & =0 \\
g^{\theta_{*} \alpha} & =-\hat{s}(r) \theta_{*} \frac{q(r)}{r} g^{\theta_{*} r}-q(r) g^{\theta_{*} \theta_{*}} \\
g^{r \alpha} & =-\hat{s}(r) \theta_{*} \frac{q(r)}{r} g^{r r}-q(r) g^{\theta_{*} r} \\
g^{\alpha \alpha} & =g^{\varphi \varphi}+q(r)^{2} g^{\theta_{*} \theta_{*}}+2 \frac{q(r)^{2} \hat{s}(r) \theta_{*}}{r} g^{\theta_{*} r}+\left[\hat{s}(r) \theta_{*}\right]^{2} \frac{q(r)^{2}}{r^{2}} g^{r r},
\end{aligned}
$$

where $\hat{s}(r)=r \partial_{r} q(r) / q(r)$ is the magnetic shear.

Writing the axisymmetric magnetic field as $\mathbf{B}=F(\psi) \nabla \varphi+\nabla \psi \times \nabla \varphi$, where $\psi$ is the poloidal flux and $F(\psi)$ is the current function, in the toric coordinate system one can write $\mathbf{B}=B_{\varphi} \nabla \varphi+\mathcal{J} B^{\theta_{*}} \nabla r \times \nabla \varphi$, while in flux-tube coordinates $\mathbf{B}=\mathcal{J} B^{\theta_{*}} \nabla r \times \nabla \alpha$, with $\mathcal{J}=\mathcal{J}_{\theta_{*} r \varphi}=\mathcal{J}_{r \alpha \theta_{*}}$. The contravariant components of the magnetic field are then given by

$$
B^{\theta_{*}}=\frac{\psi^{\prime}(r)}{\mathcal{J}}, \quad B^{r}=0, \quad B^{\varphi}=\frac{F(\psi)}{R_{c}\left(r, \theta_{*}\right)^{2}}
$$

in toric coordinates, while they write as

$$
B^{r}=B^{\alpha}=0, \quad B^{\theta_{*}}=\frac{\psi^{\prime}(r)}{\mathcal{J}}
$$

in flux-tube coordinates. Moreover, the norm of the magnetic field is computed as

$$
B\left(r, \theta_{*}\right)=\frac{F(\psi)}{R_{c}\left(r, \theta_{*}\right)} \sqrt{1+\frac{\psi^{\prime}(r)^{2}}{F(\psi)^{2}} g^{r r}} .
$$


The coefficients in Eqs. (14)-(17) are then given by

$$
\begin{aligned}
& \mathcal{P}^{y x}=\frac{b_{\varphi} a}{\mathcal{J}}, \quad \mathcal{P}^{x z}=\frac{b_{\theta_{*}}}{\mathcal{J}}, \quad \quad P^{z y}=\frac{b_{r} a}{\mathcal{J}} \\
& \mathcal{D}^{y}=a R_{0} b^{\theta_{*}}, \quad \mathcal{D}^{x}=0, \quad \mathcal{D}^{z}=R_{0} b^{\varphi} \\
& \mathcal{C}^{x}=-\frac{R_{0} B}{2 \mathcal{J}} \frac{\partial c_{\varphi}}{\partial \theta_{*}}, \mathcal{C}^{y}=\frac{a R_{0} B}{2 \mathcal{J}} \frac{\partial c_{\varphi}}{\partial r}, \quad \quad \mathcal{C}^{z}=\frac{R_{0} B}{2 \mathcal{J}}\left(\frac{\partial c_{r}}{\partial \theta_{*}}-\frac{\partial c_{\theta_{*}}}{\partial r}\right) \\
& \mathcal{N}^{x x}=g^{r r}, \quad \mathcal{N}^{x y}=2 a g^{r \theta_{*}}, \quad \mathcal{N}^{y y}=a^{2}\left[g^{\theta_{*} \theta_{*}}-\left(b^{\theta_{*}}\right)^{2}\right] \\
& \mathcal{N}^{x}=\nabla^{2} r, \quad \mathcal{N}^{z}=-\frac{1}{\mathcal{J}} \frac{\partial}{\partial \theta_{*}}\left(\mathcal{J} b^{\theta_{*}} b^{\varphi}\right), \quad \quad \mathcal{N}^{z z}=g^{\varphi \varphi}-\left(b^{\varphi}\right)^{2} \\
& \mathcal{N}^{y z}=-2 a b^{\theta_{*}} b^{\varphi}, \mathcal{N}^{y}=a\left\{\nabla^{2} \theta_{*}-\frac{1}{\mathcal{J}} \frac{\partial}{\partial \theta_{*}}\left[\mathcal{J}\left(b^{\theta_{*}}\right)^{2}\right]\right\},
\end{aligned}
$$

where $c_{i}=b_{i} / B$. Since turbulence is characterized by $\nabla_{\perp} A / \nabla_{\|} A \gg 1$, it is possible to make the following approximations when evaluating the $z$ derivatives

$$
\begin{aligned}
\frac{\partial}{\partial z} & =\frac{1}{R_{0} b^{\varphi}} \nabla_{\|}-\frac{a}{q} \frac{\partial}{\partial y} \simeq-\frac{a}{q} \frac{\partial}{\partial y} \\
\frac{\partial^{2}}{\partial y \partial z} & =\frac{1}{R_{0} b^{\varphi}} \frac{\partial}{\partial y} \nabla_{\|}-\frac{1}{a R_{0}\left(b^{\varphi}\right)^{2}} \frac{\partial b^{\varphi}}{\partial \theta_{*}} \nabla_{\|}-\frac{a}{q} \frac{\partial^{2}}{\partial y^{2}} \simeq-\frac{a}{q} \frac{\partial^{2}}{\partial y^{2}} \\
\frac{\partial^{2}}{\partial z^{2}} & =\frac{1}{\left(R_{0} b^{\varphi}\right)^{2}} \nabla_{\|}^{2}+\frac{a^{2}}{q^{2}} \frac{\partial^{2}}{\partial y^{2}}+\frac{b^{\theta_{*}}}{R_{0}\left(b^{\varphi}\right)^{3}} \frac{\partial b^{\varphi}}{\partial \theta_{*}} \nabla_{\|}-2 \frac{a b^{\theta_{*}}}{R_{0}\left(b^{\varphi}\right)^{2}} \frac{\partial}{\partial y} \nabla_{\|} \simeq \frac{a^{2}}{q^{2}} \frac{\partial^{2}}{\partial y^{2}},
\end{aligned}
$$

where we neglect the parallel derivatives with respect to the $y$ derivatives. Consequently, the coefficients of Eq. (A6) can be simplified into

$$
\begin{array}{lll}
\mathcal{P}^{y x}=\frac{a}{\mathcal{J} b_{\varphi}}, & \mathcal{P}^{x z}=P^{z y}=0 & \\
\mathcal{C}^{x}=-\frac{R_{0} B}{2 \mathcal{J}} \frac{\partial c_{\varphi}}{\partial \theta_{*}}, \mathcal{C}^{y}=\frac{a R_{0} B}{2 \mathcal{J}}\left[\frac{\partial c_{\varphi}}{\partial r}+\frac{1}{q}\left(\frac{\partial c_{\theta_{*}}}{\partial r}-\frac{\partial c_{r}}{\partial \theta_{*}}\right)\right], \mathcal{C}^{z}=0 & \mathcal{N}^{y y}=a^{2}\left(g^{\theta_{*} \theta_{*}}+\frac{g^{\varphi \varphi}}{q^{2}}\right) \\
\mathcal{N}^{x x}=g^{r r}, & \mathcal{N}^{x y}=2 a g^{r \theta_{*}}, & \mathcal{N}^{z}=0 \\
\mathcal{N}^{x}=\nabla^{2} r, & \mathcal{N}^{y}=a \nabla^{2} \theta_{*}, & \\
\mathcal{N}^{y z}=\mathcal{N}^{z z}=0 . &
\end{array}
$$


For the $(X, Y, Z)$ coordinate system, the geometric coefficients are

$$
\begin{array}{lll}
\mathcal{P}^{X Y}=-\frac{b_{\theta_{*}} a}{\mathcal{J} q}, & \mathcal{P}^{Y Z}=-\frac{a b_{r}}{\mathcal{J}}, & \mathcal{P}^{Z X}=-\frac{q b_{\alpha}}{\mathcal{J}} \\
\mathcal{D}^{X}=\mathcal{D}^{Y}=0, & \mathcal{D}^{Z}=q R_{0} b^{\theta_{*}} & \\
\mathcal{C}^{X}=-\frac{R_{0} B}{2 \mathcal{J}} \frac{\partial c_{\alpha}}{\partial \theta_{*}}, \mathcal{C}^{Y}=\frac{a R_{0} B}{2 \mathcal{J} q}\left(\frac{\partial c_{r}}{\partial \theta_{*}}-\frac{\partial c_{\theta_{*}}}{\partial r}\right), \mathcal{C}^{Z}=\frac{q R_{0} B}{2 \mathcal{J}} \frac{\partial c_{\alpha}}{\partial r} \\
\mathcal{N}^{X X}=g^{r r}, & \mathcal{N}^{X Y}=\frac{2 g^{\alpha r} a}{q}, & \mathcal{N}^{Y Y}=\frac{a^{2} g^{\alpha \alpha}}{q^{2}} \\
\mathcal{N}^{X}=\nabla^{2} r, & \mathcal{N}^{Y}=\frac{a}{q} \nabla^{2} \alpha, & \mathcal{N}^{Z}=q R_{0}\left\{\nabla^{2} \theta_{*}-\frac{1}{\mathcal{J}} \frac{\partial}{\partial \theta_{*}}\left[\mathcal{J}\left(b^{\theta_{*}}\right)^{2}\right]\right\} \\
\mathcal{N}^{Y Z}=2 a g^{\theta_{*} \alpha}, & \mathcal{N}^{X Z}=2 q g^{r \theta_{*}}, & \mathcal{N}^{Z Z}=q^{2}\left[g^{\theta_{*} \theta_{*}}-\left(b^{\theta_{*}}\right)^{2}\right] .
\end{array}
$$

Assuming $\nabla_{\perp} A / \nabla_{\|} A \gg 1$, it is possible to simplify the coefficients of Eq. (A9) and neglect the $Z$ derivatives with respect to the $X$ and $Y$ derivatives.

\section{APPENDIX B: DERIVATION OF THE MAGNETIC EQUILIBRIUM FROM THE GRAD SHAFRANOV EQUATION}

The magnetic equilibrium presented in Sec. II C is obtained by solving the Grad-Shafranov equation in the $\bar{\epsilon} \rightarrow 0$ limit, being $\bar{\epsilon}=r / R_{0}$ the local inverse aspect ratio. The main steps of the derivation are summarized here, while for a detailed discussion we refer to Ref. [30]. Note that in the remainder of this section we work in SI units. The magnetic surfaces $\left(R_{c}, Z_{c}\right)$ are modeled by

$$
\begin{aligned}
& R_{c}(r, \theta)=R_{0}\left\{1+\bar{\epsilon} \cos \theta+\frac{\Delta(r)}{R_{0}}+\sum_{m=2}^{3} \frac{S_{m}(r)}{R_{0}} \cos [(m-1) \theta]-\frac{P(r)}{R_{0}} \cos \theta\right\} \\
& Z_{c}(r, \theta)=R_{0}\left\{\bar{\epsilon} \sin \theta-\sum_{m=2}^{3} \frac{S_{m}(r)}{R_{0}} \sin [(m-1) \theta]-\frac{P(r)}{R_{0}} \sin \theta\right\}
\end{aligned}
$$

where $\Delta(r)$ is the Shafranov's shift, being $\Delta(a)=0, S_{2}(r)$ and $S_{3}(r)$ shaping coefficients related to $\kappa$ and $\delta$ according to $\kappa=\left[a-S_{2}(a)\right] /\left[a+S_{2}(a)\right]$ and $\delta=4 S_{3}(a) / a$, and $P(r)$ is a correction factor used to ensure $\psi^{\prime}(r) / F(\psi)=\bar{\epsilon} / q(r)+\mathcal{O}\left(\bar{\epsilon}^{4}\right)$. Assuming $\Delta(r) / R_{0} \sim S_{m}(r) / R_{0} \sim P(r) / r \sim \mathcal{O}\left(\bar{\epsilon}^{2}\right)$, with $m=\{2,3\}$, we expand $\mathcal{J}_{\theta r \varphi} / R_{c}^{2}=$ $\left(\partial_{r} R_{c} \partial_{\theta} Z_{c}-\partial_{\theta} R_{c} \partial_{r} Z_{c}\right) / R_{c}$ to third order in $\bar{\epsilon}$, and, imposing $\psi^{\prime}(r)=\bar{\epsilon} F(\psi) / q(r)+\mathcal{O}\left(\bar{\epsilon}^{4}\right)$, we obtain

$$
\frac{P(r)}{R_{0}}=\bar{\epsilon}\left(\frac{\bar{\epsilon}^{2}}{8}-\frac{\Delta(r)}{2 R_{0}}\right)+\sum_{m=2}^{3} \frac{1-m}{2 \bar{\epsilon}}\left[\frac{S_{m}(r)}{R_{0}}\right]^{2}+\mathcal{O}\left(\bar{\epsilon}^{4}\right) .
$$


In cylindrical coordinates $\left(R_{c}, \varphi, Z_{c}\right)$, the Grad-Shafranov operator $\Delta^{*}$ is given by

$$
\Delta^{*} \psi=R_{c}^{2} \nabla \cdot\left(\frac{\nabla \psi}{R_{c}^{2}}\right)
$$

and, using the $(\theta, r, \varphi)$ coordinate system, it can be written as

$$
\Delta^{*} \psi=\frac{R_{c}(r, \theta)^{2}}{\mathcal{J}_{\theta r \varphi}}\left[\frac{\partial}{\partial r}\left(\frac{\psi^{\prime}(r) g_{\theta \theta}}{\mathcal{J}_{\theta r \varphi}}\right)-\frac{\partial}{\partial \theta}\left(\frac{\psi^{\prime}(r) g_{r \theta}}{\mathcal{J}_{\theta r \varphi}}\right)\right] .
$$

Combining Eqs. (B1)-(B2) with Eq. (B5), expanding the result to first order in $\bar{\epsilon}$, and writing separately each one of the $m$ Fourier components, we obtain

$$
\begin{aligned}
\frac{1}{2 r^{2}}\left[r^{2} \psi^{\prime}(r)^{2}\right]^{\prime}+\mu_{0} R_{0}^{2} p^{\prime}(\psi)+F(\psi) F^{\prime}(\psi) & =0 \\
\Delta^{\prime \prime}(r)+\Delta^{\prime}(r)\left[2 \frac{\psi^{\prime \prime}(r)}{\psi^{\prime}(r)}+\frac{1}{r}\right]+\frac{1}{R_{0}}-\frac{2 R_{0} r p^{\prime}(\psi)}{\psi^{\prime}(r)^{2}} & =0 \\
S_{m}^{\prime \prime}(r)+\left[2 \frac{\psi^{\prime \prime}(r)}{\psi^{\prime}(r)}+\frac{1}{r}\right] S_{m}^{\prime}(r)+\frac{1-m^{2}}{r} S_{m} & =0,
\end{aligned}
$$

where $p(\psi)$ is the plasma pressure at the flux surface $\psi$. Assuming $F(r)=B_{0} R_{0}\left[1+F_{2}(r)\right]$, where $F_{2}(r) \sim \bar{\epsilon}^{2}$, and expanding Eq. (B6) to first order in $\bar{\epsilon}$, we obtain

$$
\begin{array}{r}
F_{2}^{\prime}(r)=\mu_{0} \frac{p^{\prime}(r)}{B_{0}^{2}}-\frac{r}{R_{0}^{2}} \frac{2-\hat{s}(r)}{q(r)^{2}} \\
\Delta^{\prime}(r)=-\bar{\epsilon}\left(\beta_{p}(r)+\frac{l_{i}(r)}{2}\right) \\
r^{2} S_{m}^{\prime \prime}(r)+r[3-2 \hat{s}(r)] S_{m}^{\prime}(r)+\left(1-m^{2}\right) S_{m}=0,
\end{array}
$$

with

$$
l_{i}(r)=\frac{2 q(r)^{2}}{r^{4}} \int_{0}^{r} \frac{\bar{r}^{3}}{q(\bar{r})^{2}} \mathrm{~d} \bar{r}, \quad \quad \beta_{p}(r)=-\frac{2 \mu_{0} R_{0}^{2} q(r)^{2}}{r^{4} B_{0}^{2}} \int_{0}^{r} p^{\prime}(\bar{r}) \bar{r}^{2} \mathrm{~d} \bar{r} .
$$

Equation (B7) is solved assuming a safety factor with parabolic profile and neglecting the plasma pressure contribution, i.e, assuming $q(r)=q_{0}+\left(q-q_{0}\right)(r / a)^{2}$ and $p^{\prime}(r)=0$. This gives

$$
\begin{array}{r}
F_{2}(r)=-\frac{r^{2}}{q(r)^{2} R_{0}^{2}}\left[2+\frac{q-q_{0}}{q_{0}}\left(\frac{r}{a}\right)^{2}\right] \\
l_{i}(r)=\frac{a^{2} q(r)^{2}}{r^{2}\left(q-q_{0}\right)}\left[\frac{a^{2}}{r^{2}\left(q-q_{0}\right)} \log \left(\frac{q(r)}{q_{0}}\right)-\frac{1}{q(r)}\right] \\
S_{m}(r)=S_{m}(a)\left(\frac{r}{a}\right)^{m-1} \frac{q(r) \hat{s}(r)+2 q_{0} \frac{m+1}{m-1}}{q \hat{s}+2 q_{0} \frac{m+1}{m-1}} .
\end{array}
$$


The Shafranov's shift is therefore

$$
\Delta(r)=-\frac{1}{2 R_{0}} \int_{a}^{r} \bar{r} l_{i}(\bar{r}) \mathrm{d} \bar{r}
$$

Equations (B1)-(B3), (B9)-(B10), and (28)-(29) define the magnetic equilibrium used in the present paper.

\section{APPENDIX C: DERIVATION OF THE CURVATURE OPERATOR FOR $\epsilon=0$}

To obtain an analytical expression for the curvature operator, we simplify the $\mathcal{C}^{Y}$ coefficient as follows. First, we assume $\mathcal{C}^{Y} \simeq-\left.\partial_{r} R_{c}(r, \theta)\right|_{\theta_{*}}$, according to the results of a numerical investigation we carried out to study the leading order term in the curvature operator. Second, having observed that BMs are mainly destabilized at $\theta_{*}=0$, as discussed in Sec. III, we assume $\mathcal{C}^{Y} \simeq-\left.\partial_{r} R_{c}(r, \theta)\right|_{\theta_{*}=0}$. Third, combining Eq. (B1) with Eq. (B3), expanding the result in $\epsilon$, and retaining zeroth and first order terms only, we write

$$
R_{c}(r, \theta)=R_{0}\left\{1+\frac{r}{R_{0}} \cos \theta+\sum_{m=2}^{3} \frac{S_{m}(r)}{R_{0}} \cos [(m-1) \theta]-\sum_{m=2}^{3} \frac{1-m}{2 r R_{0}}\left[S_{m}(r)\right]^{2} \cos \theta\right\} .
$$

Fourth, expressing $\left.\partial_{r} R_{c}(r, \theta)\right|_{\theta_{*}=0}=\partial_{r} R_{c}(r, 0)+\left.\partial_{\theta} R_{c}(r, 0) \partial_{r} \theta\right|_{\theta_{*}=0}$ and noting that $\left.\partial_{r} \theta\right|_{\theta_{*}=0}=$ 0 for $\epsilon=0$, we obtain

$$
\left.\partial_{r} R_{c}(r, \theta)\right|_{\theta_{*}=0}=1+\sum_{m=2}^{3} S_{m}^{\prime}(a)-\sum_{m=2}^{3} \frac{1-m}{a}\left[S_{m}(a) S_{m}^{\prime}(a)-\frac{S_{m}(a)^{2}}{2 a}\right] .
$$

Finally, substituting the $S_{m}(r)$ expression given in Eq. (B9) into Eq. (C2) and using the definition of $\kappa$ and $\delta$, we deduce

$$
\left.\partial_{r} R_{c}(r, \theta)\right|_{\theta_{*}=0}=1+\frac{\delta q}{1+q}+\frac{\delta^{2}(7 q-1)}{16(1+q)}-\frac{\kappa-1}{2(\kappa+1)}-\frac{(\kappa-1)(5 q-2)}{(\kappa+1)^{2}(2+q)} .
$$

This is the result presented in Eq. (48).

[1] A. Loarte, B. Lipschultz, A. S. Kukushkin, G. F. Matthews, P. C. Stangeby, N. Asakura, G. F. Counsell, G. Federici, A. Kallenbach, K. Krieger, A. Mahdavi, V. Philipps, D. Reiter, J. Roth, J. Strachan, D. Whyte, R. Doerner, T. Eich, W. Fundamenski, A. Herrmann, M. Fenstermacher, P. Ghendrih, M. Groth, A. Kirschner, S. Konoshima, B. LaBombard, P. Lang, 
A. W. Leonard, P. Monier-Garbet, R. Neu, H. Pacher, B. Pegourie, R. A. Pitts, S. Takamura, J. Terry, E. Tsitrone, the Itpa Scrape-off Layer Group, and Diver. Chapter 4: Power and particle control. Nuclear Fusion, 47(6):S203-S263, jun 2007.

[2] B. Scott. Three-dimensional computation of drift Alfvén turbulence. Plasma Physics and Controlled Fusion, 39(10):1635-1668, oct 1997.

[3] J. W. Connor, R. J. Hastie, H. R. Wilson, and R. L. Miller. Magnetohydrodynamic stability of tokamak edge plasmas. Physics of Plasmas, 5(7):2687, 1998.

[4] A. Zeiler. 3D fluid simulations of Tokamak edge turbulence. IPP Report 6 333, Max-PlanckInstitut fur Plasmaphysik, 1999.

[5] J. R. Myra, D. A. D'Ippolito, X. Q. Xu, and R. H. Cohen. Resistive modes in the edge and scrape-off layer of diverted tokamaks. Physics of Plasmas, 7(11):4622, 2000.

[6] D. D. Ryutov and R. H. Cohen. Instability Driven by Sheath Boundary Conditions and Limited to Divertor Legs. Contributions to Plasma Physics, 44(13):168-175, apr 2004.

[7] P. Ricci. Simulation of the scrape-off layer region of tokamak devices. Journal of Plasma Physics, 81(02):435810202, apr 2015.

[8] Y. Sarazin, Ph. Ghendrih, G. Attuel, C. Clément, X. Garbet, V. Grandgirard, M. Ottaviani, S. Benkadda, P. Beyer, N. Bian, and C. Figarella. Theoretical understanding of turbulent transport in the SOL. Journal of Nuclear Materials, 313-316(SUPPL.):796-803, mar 2003.

[9] O. E. Garcia, V. Naulin, A. H. Nielsen, and J. Juul Rasmussen. Computations of Intermittent Transport in Scrape-Off Layer Plasmas. Physical Review Letters, 92(16):165003, apr 2004.

[10] J. R. Myra, D. A. Russell, D. A. D'Ippolito, J.-W. Ahn, R. Maingi, R. J. Maqueda, D. P. Lundberg, D. P. Stotler, S. J. Zweben, J. Boedo, M. Umansky, and NSTX Team. Reduced model simulations of the scrape-off-layer heat-flux width and comparison with experiment. Physics of Plasmas, 18(1):012305, 2011.

[11] T. T. Ribeiro and B. Scott. Gyrofluid turbulence studies of the effect of the poloidal position of an axisymmetric Debye sheath. Plasma Physics and Controlled Fusion, 50(5):055007, may 2008 .

[12] B. D. Dudson, M. V. Umansky, X. Q. Xu, P. B. Snyder, and H. R. Wilson. BOUT++: A framework for parallel plasma fluid simulations. Computer Physics Communications, 180(9):14671480, sep 2009.

[13] P. Tamain, Ph. Ghendrih, H. Bufferand, G. Ciraolo, C. Colin, N. Fedorczak, N. Nace, 
F. Schwander, and E. Serre. Multi-scale self-organisation of edge plasma turbulent transport in 3D global simulations. Plasma Physics and Controlled Fusion, 57(5):054014, may 2015.

[14] A. H. Nielsen, G. S. Xu, J. Madsen, V. Naulin, J. Juul Rasmussen, and B. N. Wan. Simulation of transition dynamics to high confinement in fusion plasmas. Physics Letters A, 379(4748):3097-3101, dec 2015.

[15] P. Ricci, F. D. Halpern, S. Jolliet, J. Loizu, A. Mosetto, A. Fasoli, I. Furno, and C. Theiler. Simulation of plasma turbulence in scrape-off layer conditions: the GBS code, simulation results and code validation. Plasma Physics and Controlled Fusion, 54(12):124047, dec 2012.

[16] F. D. Halpern, P. Ricci, S. Jolliet, J. Loizu, J. Morales, A. Mosetto, F. Musil, F. Riva, T. M. Tran, and C. Wersal. The GBS code for tokamak scrape-off layer simulations. Journal of Computational Physics, 315:388-408, jun 2016.

[17] A. Mosetto, F. D. Halpern, S. Jolliet, J. Loizu, and P. Ricci. Turbulent regimes in the tokamak scrape-off layer. Physics of Plasmas, 20(9):092308, 2013.

[18] S. Jolliet, F. D. Halpern, J. Loizu, A. Mosetto, and P. Ricci. Aspect ratio effects on limited scrape-off layer plasma turbulence. Physics of Plasmas, 21(2):022303, feb 2014.

[19] A. Mosetto, F. D. Halpern, S. Jolliet, J. Loizu, and P. Ricci. Finite ion temperature effects on scrape-off layer turbulence. Physics of Plasmas, 22(1):012308, jan 2015.

[20] F. D. Halpern, P. Ricci, B. Labit, I. Furno, S. Jolliet, J. Loizu, A. Mosetto, G. Arnoux, J. P. Gunn, J. Horacek, M. Kočan, B. LaBombard, and C. Silva. Theory-based scaling of the SOL width in circular limited tokamak plasmas. Nuclear Fusion, 53(12):122001, dec 2013.

[21] F. D. Halpern, P. Ricci, S. Jolliet, J. Loizu, and A. Mosetto. Theory of the scrape-off layer width in inner-wall limited tokamak plasmas. Nuclear Fusion, 54(4):043003, apr 2014.

[22] Y. Camenen, A. Pochelon, R. Behn, A. Bottino, A. Bortolon, S. Coda, A. Karpushov, O. Sauter, G. Zhuang, and the TCV Team. Impact of plasma triangularity and collisionality on electron heat transport in TCV L-mode plasmas. Nuclear Fusion, 47(7):510-516, jul 2007.

[23] J. E. Kinsey, R. E. Waltz, and J. Candy. The effect of plasma shaping on turbulent transport and ExB shear quenching in nonlinear gyrokinetic simulations. Physics of Plasmas, 14(10):102306, 2007.

[24] E. A. Belli, G. W. Hammett, and W. Dorland. Effects of plasma shaping on nonlinear gyroki- 
netic turbulence. Physics of Plasmas, 15(9):092303, 2008.

[25] A. Marinoni, S. Brunner, Y. Camenen, S. Coda, J. P. Graves, X. Lapillonne, A. Pochelon, O. Sauter, and L. Villard. The effect of plasma triangularity on turbulent transport: modeling TCV experiments by linear and non-linear gyrokinetic simulations. Plasma Physics and Controlled Fusion, 51(5):055016, may 2009.

[26] Weigang Wan, Yang Chen, Scott E Parker, and Richard J Groebner. Effects of the magnetic equilibrium on gyrokinetic simulations of tokamak microinstabilities. Physics of Plasmas, 22(6):062502, jun 2015.

[27] J Horacek, R A Pitts, J Adamek, G Arnoux, J-G Bak, S Brezinsek, M Dimitrova, R J Goldston, J P Gunn, J Havlicek, S-H Hong, F Janky, B LaBombard, S Marsen, G Maddaluno, L Nie, V Pericoli, Tsv Popov, R Panek, D Rudakov, J Seidl, D S Seo, M Shimada, C Silva, P C Stangeby, B Viola, P Vondracek, H Wang, G S Xu, and Y Xu. Multi-machine scaling of the main SOL parallel heat flux width in tokamak limiter plasmas. Plasma Physics and Controlled Fusion, 58(7):074005, jul 2016.

[28] S. I. Braginskii. Transport Processes in a Plasma. Reviews of Plasma Physics, 1:205, 1965.

[29] A. Zeiler, J. F. Drake, and B. Rogers. Nonlinear reduced Braginskii equations with ion thermal dynamics in toroidal plasma. Physics of Plasmas, 4(6):2134, 1997.

[30] J. P. Graves. Toroidal drift precession and wave-particle interaction in shaped tokamaks with finite beta and neoclassical equilibrium effects. Plasma Physics and Controlled Fusion, 55(7):074009, jul 2013.

[31] P. Ricci and B. N. Rogers. Plasma turbulence in the scrape-off layer of tokamak devices. Physics of Plasmas, 20(1):010702, 2013.

[32] J. Loizu, P. Ricci, F. D. Halpern, and S. Jolliet. Boundary conditions for plasma fluid models at the magnetic presheath entrance. Physics of Plasmas, 19(12):122307, 2012.

[33] J. Loizu, P. Ricci, F. D. Halpern, S. Jolliet, and A. Mosetto. On the electrostatic potential in the scrape-off layer of magnetic confinement devices. Plasma Physics and Controlled Fusion, $55(12): 124019, \operatorname{dec} 2013$.

[34] J. Loizu, P. Ricci, F. D. Halpern, S. Jolliet, and A. Mosetto. Intrinsic toroidal rotation in the scrape-off layer of tokamaks. Physics of Plasmas, 21(6):062309, jun 2014.

[35] J. Loizu, P. Ricci, F. D. Halpern, S. Jolliet, and A. Mosetto. Effect of the limiter position on the scrape-off layer width, radial electric field and intrinsic flows. Nuclear Fusion, 54(8):083033, 
$\operatorname{aug} 2014$.

[36] F. Riva, P. Ricci, F. D. Halpern, S. Jolliet, J. Loizu, and A. Mosetto. Verification methodology for plasma simulations and application to a scrape-off layer turbulence code. Physics of Plasmas, 21(6):062301, jun 2014.

[37] P. Ricci, F. Riva, C. Theiler, A. Fasoli, I. Furno, F. D. Halpern, and J. Loizu. Approaching the investigation of plasma turbulence through a rigorous verification and validation procedure: A practical example. Physics of Plasmas, 22(5):055704, may 2015.

[38] F. D. Halpern, J. L. Terry, S. J. Zweben, B. LaBombard, M. Podesta, and P. Ricci. Comparison of 3D flux-driven scrape-off layer turbulence simulations with gas-puff imaging of Alcator CMod inner-wall limited discharges. Plasma Physics and Controlled Fusion, 57(5):054005, may 2015.

[39] F. Riva, C. Colin, J. Denis, L. Easy, I. Furno, J. Madsen, F. Militello, V. Naulin, A. H. Nielsen, J. M. B. Olsen, J. T. Omotani, J. J. Rasmussen, P. Ricci, E. Serre, P. Tamain, and C. Theiler. Blob dynamics in the TORPEX experiment: a multi-code validation. Plasma Physics and Controlled Fusion, 58(4):044005, apr 2016.

[40] V. Naulin. Electromagnetic transport components and sheared flows in drift-Alfvén turbulence. Physics of Plasmas, 10(10):4016, 2003.

[41] S. J. Zweben, J. A. Boedo, O. Grulke, C. Hidalgo, B. LaBombard, R. J. Maqueda, P. Scarin, and J. L. Terry. Edge turbulence measurements in toroidal fusion devices. Plasma Physics and Controlled Fusion, 49(7):S1-S23, jul 2007.

[42] G. Q. Yu, S. I. Krasheninnikov, and P. N. Guzdar. Two-dimensional modelling of blob dynamics in tokamak edge plasmas. Physics of Plasmas, 13(4):042508, 2006.

[43] K. Bodi, G. Ciraolo, P. Ghendrih, F. Schwander, E. Serre, and P. Tamain. In 38th EPS Conference on Plasma Physics, Strasbourg, France, 2011.

[44] D. A. Russell, D. A. D'Ippolito, and J. R. Myra. On relaxing the Boussinesq approximation in scrape-off layer turbulence (SOLT) model simulations. Bulletin of the American Physical Society, 54th Annual Meeting of the APS Division of Plasma Physics, 57(12), 2012.

[45] F. D. Halpern, S. Jolliet, J. Loizu, A. Mosetto, and P. Ricci. Ideal ballooning modes in the tokamak scrape-off layer. Physics of Plasmas, 20(5):052306, 2013.

[46] B. N. Rogers and W. Dorland. Noncurvature-driven modes in a transport barrier. Physics of Plasmas, 12(6):062511, 2005. 
[47] P. Ricci, B. N. Rogers, and S. Brunner. High- and Low-Confinement Modes in Simple Magnetized Toroidal Plasmas. Physical Review Letters, 100(22):225002, jun 2008.

[48] A. Mosetto, F. D. Halpern, S. Jolliet, and P. Ricci. Low-frequency linear-mode regimes in the tokamak scrape-off layer. Physics of Plasmas, 19(11):112103, 2012.

[49] J. W. Connor, R. J. Hastie, and J. B. Taylor. Shear, Periodicity, and Plasma Ballooning Modes. Physical Review Letters, 40(6):396-399, feb 1978.

[50] G. Bateman and D. B. Nelson. Resistive-Ballooning-Mode Equation. Physical Review Letters, 41(26):1804-1807, dec 1978.

[51] D. R. McCarthy, P. N. Guzdar, J. F. Drake, T. M. Antonsen, and A. B. Hassam. Stability of resistive and ideal ballooning modes in the Texas Experimental Tokamak and DIII-D. Physics of Fluids B: Plasma Physics, 4(7):1846, 1992.

[52] S. V. Novakovskii, P. N. Guzdar, J. F. Drake, C. S. Liu, and F. L. Waelbroeck. New unstable branch of drift resistive ballooning modes in tokamaks. Physics of Plasmas, 2(3):781, 1995.

[53] T. Rafiq, G. Bateman, A. H. Kritz, and A. Y. Pankin. Development of drift-resistive-inertial ballooning transport model for tokamak edge plasmas. Physics of Plasmas, 17(8):082511, 2010.

[54] K. Mima and A. Hasegawa. Nonlinear instability of electromagnetic drift waves. Physics of Fluids, 21(1):81, 1978.

[55] M. Wakatani and A. Hasegawa. A collisional drift wave description of plasma edge turbulence. Physics of Fluids, 27(3):611, 1984.

[56] H. Sugama, M. Wakatani, and A. Hasegawa. Study of resistive drift and resistive interchange modes in a cylindrical plasma with magnetic shear. Physics of Fluids, 31(6):1601, 1988.

[57] W. Horton. Drift waves and transport. Reviews of Modern Physics, 71(3):735-778, apr 1999.

[58] P. H. Diamond, A. Hasegawa, and K. Mima. Vorticity dynamics, drift wave turbulence, and zonal flows: a look back and a look ahead. Plasma Physics and Controlled Fusion, 53(12):124001, dec 2011.

[59] B. N. Rogers and P. Ricci. Low-Frequency Turbulence in a Linear Magnetized Plasma. Physical Review Letters, 104(22):225002, jun 2010.

[60] P. Ricci and B. N. Rogers. Transport scaling in interchange-driven toroidal plasmas. Physics of Plasmas, 16(6):062303, 2009.

[61] P. Ricci and B. N. Rogers. Turbulence Phase Space in Simple Magnetized Toroidal Plasmas. 
Physical Review Letters, 104(14):145001, apr 2010.

[62] F Militello, N R Walkden, T Farley, W A Gracias, J Olsen, F Riva, L Easy, N Fedorczak, I Lupelli, J Madsen, A H Nielsen, P Ricci, P Tamain, and J Young. Multi-code analysis of scrape-off layer filament dynamics in MAST. Plasma Physics and Controlled Fusion, 58(10):105002, nov 2016.

[63] A. Arakawa. Computational design for long-term numerical integration of the equations of fluid motion: Two-dimensional incompressible flow. Part I. Journal of Computational Physics, 1(1):119-143, aug 1966.

[64] B. D. Scott. Free-energy conservation in local gyrofluid models. Physics of Plasmas, 12(10):102307, 2005. 